\title{
NUMERICAL SIMULATIONS OF AN ENERGY-TRANSPORT MODEL FOR PARTIALLY QUANTIZED PARTICLES*
}

\author{
PAOLA PIETRA ${ }^{\dagger}$ AND NICOLAS VAUCHELET ${ }^{\ddagger}$
}

\begin{abstract}
A coupled quantum-classical model describing the transport of electrons confined in nanoscale semiconductor devices is considered. Using the subband decomposition approach allows us to separate the transport directions from the confinement direction. The motion of the gas in the transport direction is assumed to be classical. Then a hierarchy of adiabatic quantum-classical models is obtained, leading to subband SHE and energy-transport models with explicit expression of the diffusion coefficients. The energy-transport-Schrödinger-Poisson model is then used for the numerical simulation of the transport of the electron gas in an ultra-scaled Double-Gate-MOSFET.
\end{abstract}

Key words. Schrödinger equation, energy-transport system, subband model, nanotransistor, Gummel iterations, mixed finite elements.

AMS subject classifications. 65M60, 65Z05, 82D37, 82D80, 35J10, 76P05.

\section{Introduction}

In nanoscale semiconductor devices, electrons might be extremely confined in one or several directions, referred to as the confining direction. This leads to a partial quantization of the energy which can be modelled by the subband decomposition method [33]. This subband decomposition approach allows one to separate the confinement direction from the transport direction. Thanks to the reduction of the dimension of the transport problem, the computational gain is significant. In the confined direction electrons behave like waves; the system is at thermodynamical equilibrium and is described by a statistical mixture of eigenstates of a Schrödinger-Poisson system. In the transport direction the transport can be of classical $[8,7]$ or quantum [33] nature.

Here, we are interested in deriving adiabatic quantum-classical models accounting for thermal effects, aiming at accurate and efficient numerical simulation of confined devices. In [9] several spherical harmonic expansion (SHE) models incorporating quantum effects are proposed. However, with their strategy the obtained models have a complicated non-local structure which is not suitable for numerical purposes. Quantum energy-transport and quantum drift-diffusion models have been derived in [16] using the strategy of quantum moments, as well as in [26]. These models involve a quantum chemical potential that depends on the density in a non-local way. In those references, fully quantum diffusive/hydrodynamic models are derived and then expanded semiclassically (typically up to $O\left(\hbar^{2}\right)$ terms). In this work we deal with a completely quantum description of the (quantizied) energy in the transverse direction, and with completely (semi)classical description in the transport directions. This in particular means that the considered time and space scales allow us to consider both the transport equation and the collision description in the classical setting. Possible memory effects for the electron-phonon interaction reported in the literature [19, 36]

${ }^{*}$ Received: June 26, 2012; accepted (in revised form): December 11, 2012. Communicated by Pierre Degond.

$\dagger$ Istituto di Matematica Applicata e Tecnologie Informatiche "Enrico Magenes", CNR, Via Ferrata 1, 27100 Pavia, Italy (paola.pietra@imati.cnr.it).

${ }^{\ddagger}$ UPMC Univ Paris 06, UMR 7598, Laboratoire Jacques-Louis Lions, F-75005, Paris, France CNRS, UMR 7598, Laboratoire Jacques-Louis Lions, F-75005, Paris, France (vauchelet@ann.jussieu. fr). 
are not taken into account here. We follow the strategy proposed in [7] where the subband model is derived first, and then a diffusive approximation of the adiabatic Boltzmann equation is performed to obtain coupled quantum-fluid models (spherical harmonic expansion and energy-transport). More precisely, the adiabatic Boltzmann equation for semiconductors governs the evolution of the distribution function $f_{n}(t, x, k)$ of the $n$th subband, denoted by $\epsilon_{n}$ :

$$
\partial_{t} f_{n}+\frac{\hbar^{2}}{m^{*}} k \cdot \nabla_{x} f_{n}-\nabla_{x} \epsilon_{n} \cdot \nabla_{k} f_{n}=Q(f)_{n},
$$

where the collision operator $Q$ accounts for collisions in the subband, as well as for transitions between subbands, and considers different kind of collision mechanisms (collisions with impurities, collisions with phonons and electron-electron collisions). The diffusive approximation is obtained by assuming that these scattering mechanisms are predominant. The subband energy-transport (ET) model in [7], directly derived from the Boltzmann equations (as in e.g. [6] for the classical case), is, however, not immediately suited for numerical simulations, since the diffusion coefficients are not given in explicit form and, moreover, the energy relaxation term is not obtained. Therefore, we propose in this work a suitable description of the dominant collision mechanisms which allows us to extend the formal derivation of the SHE model given in [7]. Assuming that the transport is mainly driven by the elastic collisions, a diffusive approximation of the above Boltzmann equation allows us to derive the SHE model in the confined case:

$$
N \partial_{t} F+\nabla_{x} \cdot J-\kappa \partial_{\varepsilon} F=S(F)
$$

where $N$ is the density of states, $F$ is the formal limit of the distribution function, $J=$ $-D \nabla_{x} F$ is the electronic current, and $S$ is the scattering operator. The confinement in the transverse direction is taken into account through the expressions of the density of states, the coefficient $\kappa$, and the diffusion coefficient $D$. Then a new ET model is formally derived as diffusive limit from this SHE model (in the spirit of [3]) i.e. assuming the predominance of electron-electron scattering. This ET system governs the evolution of the particles density $\rho$ and of the energy density $\rho \mathcal{E}$, which are defined through the quasi-Fermi potential energy $\mu$ and the temperature $T$ of the system by the Fermi-Dirac statistics $\mathcal{F}_{\mu, T}$. More precisely,

$$
\rho=\int_{\mathbb{R}} N(\varepsilon) \mathcal{F}_{\mu, T}(\varepsilon) d \varepsilon, \quad \rho \mathcal{E}=\int_{\mathbb{R}} \varepsilon N(\varepsilon) \mathcal{F}_{\mu, T}(\varepsilon) d \varepsilon .
$$

The ET system for partially confined particles writes

$$
\begin{gathered}
\partial_{t} \rho-\nabla_{x} \cdot\left(\mathbb{D}_{00} \nabla_{x}\left(\frac{\mu}{k_{B} T}\right)-\mathbb{D}_{01} \nabla_{x}\left(\frac{1}{k_{B} T}\right)\right)=0 \\
\partial_{t} \rho \mathcal{E}+\sum_{n \in \mathbb{N}^{*}} \frac{2 \pi m^{*}}{\hbar^{2}} \partial_{t} \epsilon_{n} \int_{\epsilon_{n}}^{+\infty} \mathcal{F}_{\mu, T}(\varepsilon) d \varepsilon-\nabla_{x} \cdot\left(\mathbb{D}_{10} \nabla_{x}\left(\frac{\mu}{k_{B} T}\right)-\mathbb{D}_{11} \nabla_{x}\left(\frac{1}{k_{B} T}\right)\right)=W
\end{gathered}
$$

where $\mathbb{D}$ is a diffusion matrix, incorporating the quantum confinement in the transverse direction, and $W$ is the relaxation term. The Boltzmann constant is denoted by $k_{B}$.

Numerical discretization of classical ET equations has already been studied in many papers: by using mixed finite elements schemes e.g. in [15, 20, 21, 23, 28, 30], 
ENO schemes in [24], finite difference methods [18, 35], and finite volume schemes in [13]. In [15, 23], the authors propose a drift-diffusion reformulation which allows one to use an accurate Sharfetter-Gummel scheme with exponential fitting [11] and, moreover, to decouple the ET model. However, in this quantum case, the involved form of the diffusion coefficients does not allow for a decoupled drift-diffusion reformulation. Then we will use a more traditional approach with mixed finite elements, which can be directly applied since the obtained ET model turns out to be in symmetric form. Then, a Gummel type algorithm is used as outer iterations of the solution of the coupled energy-transport-Schrödinger-Poisson model, and the (non-linear) ET discrete system is solved by means of a Newton scheme. Moreover, passing to the limit in the energy relaxation term, a subband drift-diffusion equation is recovered in the form of [8] with a more accurate description of the diffusion coefficients taking into account the collisional mechanisms (see also [32] for numerical simulations).

The outline of the paper is the following. In Section 2 we set the assumptions on the collision mechanisms and we briefly present the formal derivation of the SHE model. Then, we derive from this latter model the novel subband ET model. Finally, a subband drift-diffusion equation is obtained in the limit when the relaxation time goes to infinity. Section 3 is devoted to the numerical issues. Subsection 3.1 presents the complete stationary model, the mixed finite elements scheme is described in Subsection 3.2, and the iterative approach is outlined in Subsection 3.3. Numerical simulations of an ultra-scaled Double-Gate MOSFET are presented in Subsection 3.4.

\section{Formal derivation of adiabatic fluid-quantum models}

2.1. The quantum-kinetic framework. We will assume in this work that the confinement direction is one dimensional whereas the transport takes place in a two dimensional domain. The domain is denoted $\Omega=\omega \times[0, \ell]$ with $\omega \subset \mathbb{R}^{2}$. The first two directions, called $x \in \omega$, correspond to the classical description of the gas, whereas in the third direction $z \in[0, \ell]$ quantum effects occur. The quantum confinement of the electron gas is described thanks to the eigen-elements of the 1D Schrödinger operator. They are denoted $\left(\epsilon_{n}, \chi_{n}\right)_{n \in \mathbb{N}^{*}}$ and solve the following eigenvalue problem:

$$
\left\{\begin{array}{l}
-\frac{\hbar^{2}}{2} \frac{d}{d z}\left(\frac{1}{m^{*}} \frac{d}{d z} \chi_{n}\right)+\left(U+U_{c}\right) \chi_{n}=\epsilon_{n} \chi_{n}, \\
\chi_{n}(x, \cdot) \in H_{0}^{1}(0, \ell), \quad \int_{0}^{\ell} \chi_{n} \chi_{n^{\prime}} d z=\delta_{n n^{\prime}} .
\end{array}\right.
$$

In this equation $\hbar$ is the reduced Planck constant, $m^{*}$ the effective mass, and $U_{c}$ is a given confining potential. It is known that the eigenvalues $\epsilon_{n}$ in (2.1) form an increasing sequence tending to $+\infty$. These functions depend on the potential energy defined by $U=-e V$, where $e$ is the elementary charge and $V$ denotes the self-consistent electrostatic potential which solves the Poisson equation

$$
\operatorname{div}_{x, z}\left(\varepsilon_{R}(x, z) \nabla_{x, z} V\right)=\frac{e}{\varepsilon_{0}}\left(N_{e}-N_{D}\right) .
$$

Here $\varepsilon_{R}(x, z)$ denotes the relative permittivity, $\varepsilon_{0}$ the permittivity constant in vacuum, $N_{D}(x, z)$ is the prescribed doping density, and $N_{e}(t, x, z)$ is the electron density. This density is described by a sequence of distribution functions $\left(f_{n}\right)_{n \in \mathbb{N}^{*}}$ describing the repartition on each subband for the classical direction $x \in \omega$ and the corresponding momentum variable $k \in \mathbb{R}^{2}$. It is written as

$$
N_{e}(t, x, z)=\sum_{n=1}^{+\infty}\left(\int_{\mathbb{R}^{2}} f_{n}(t, x, k) d k\right)\left|\chi_{n}\right|^{2}(t, x, z) .
$$


The evolution of distribution functions is governed by a classical transport model in the $x$ direction parallel to the gas. The total energy of the $n$th subband is defined by

$$
\varepsilon_{n}(t, x, k)=\frac{|k|^{2} \hbar^{2}}{2 m^{*}}+\epsilon_{n}(t, x) .
$$

Therefore the energy-band diagram of the semiconductor crystal is spherically symmetric and strictly monotone with respect to $|k|$. Then the Brillouin zone (which represents the elementary cell of the dual lattice $L^{*}$ ) is equal to $\mathbb{R}^{2}$. Moreover, we point out that, in contrast with the classical counterpart, the energy-band depends on space and time. In a kinetic collisional framework, the distribution function $f_{n}$ of the $n$th subband satisfies the rescaled Boltzmann transport equation [4, 32]:

$$
\alpha^{2} \partial_{t} f_{n}^{\alpha}+\alpha\left(\nabla_{k} \varepsilon_{n} \cdot \nabla_{x} f_{n}^{\alpha}-\nabla_{x} \varepsilon_{n} \cdot \nabla_{k} f_{n}^{\alpha}\right)=Q_{l d}\left(f^{\alpha}\right)_{n}+\frac{\alpha^{2}}{\beta} Q_{e}\left(f^{\alpha}\right)_{n},
$$

where $Q_{l d}$ is the collision operator for the lattice defect collisions and $Q_{e}$ is the collision operator for the elastic, nonlinear electron-electron collisions, accounting for intraband scattering as well as for transitions between subbands. $\alpha$ and $\beta$ are dimensionless parameters that satisfy $\alpha \ll \beta \ll 1$.

The main classes of lattice-defects that we shall consider are impurities and phonons [1]:

$$
Q_{l d}(f)=Q_{i m p}(f)+Q_{p h}(f) .
$$

The elastic character of the impurity scattering leads to

$$
Q_{i m p}(f)_{n}(k):=\sum_{n^{\prime} \in \mathbb{N}^{*}} \int_{\mathbb{R}^{2}} \Phi_{n, n^{\prime}}^{i m p}\left(k, k^{\prime}\right) \delta\left(\varepsilon_{n}(k)-\varepsilon_{n^{\prime}}\left(k^{\prime}\right)\right)\left(f_{n^{\prime}}\left(t, x, k^{\prime}\right)-f_{n}(t, x, k)\right) d k^{\prime},
$$

where $\delta$ is the Dirac measure and the dependence on $t, x$ of $\varepsilon$ and $\Phi^{i m p}$ has been omitted. The cross-section is assumed to be symmetric: $\Phi_{n, n^{\prime}}^{i m p}\left(k, k^{\prime}\right)=\Phi_{n^{\prime}, n}^{i m p}\left(k^{\prime}, k\right)$. The electron-phonon collision operator is considered as

$$
\begin{aligned}
& Q_{p h}(f)_{n}(k) \\
& =\sum_{n^{\prime} \in \mathbb{N}^{*}} \int_{\mathbb{R}^{2}} \Phi_{n, n^{\prime}}^{p h}\left(k, k^{\prime}\right)\left(\left[\left(N_{p h}+1\right) \delta\left(\varepsilon_{n}(k)-\varepsilon_{n^{\prime}}\left(k^{\prime}\right)+\alpha^{2} \varepsilon_{p h}\right)\right.\right. \\
& \left.\quad+N_{p h} \delta\left(\varepsilon_{n}(k)-\varepsilon_{n^{\prime}}\left(k^{\prime}\right)-\alpha^{2} \varepsilon_{p h}\right)\right] f_{n^{\prime}}\left(k^{\prime}\right)\left(1-\eta f_{n}(k)\right) \\
& \quad-\left[\left(N_{p h}+1\right) \delta\left(\varepsilon_{n^{\prime}}\left(k^{\prime}\right)-\varepsilon_{n}(k)+\alpha^{2} \varepsilon_{p h}\right)\right. \\
& \left.\left.\quad+N_{p h} \delta\left(\varepsilon_{n^{\prime}}\left(k^{\prime}\right)-\varepsilon_{n}(k)-\alpha^{2} \varepsilon_{p h}\right)\right] f_{n}(k)\left(1-\eta f_{n^{\prime}}\left(k^{\prime}\right)\right)\right) d k^{\prime},
\end{aligned}
$$

where again $\Phi_{n, n^{\prime}}^{p h}\left(k, k^{\prime}\right)=\Phi_{n^{\prime}, n}^{p h}\left(k^{\prime}, k\right), \varepsilon_{p h}$ is the phonon energy, $\eta \geq 0$ is a dimensionless distribution function scaling factor, and the terms $0 \leq 1-\eta f_{n} \leq 1$ express the Pauli exclusion principle. $N_{p h}$ is the phonon occupation number, given by the Bose-Einstein statistics

$$
N_{p h}=\left(e^{\alpha^{2} \varepsilon_{p h} /\left(k_{B} T_{L}\right)}-1\right)^{-1}
$$


with $T_{L}$ the lattice temperature and $k_{B}$ the Boltzmann constant. Formally expanding the phonon collision operator in powers of $\alpha^{2}$, we get

$$
Q_{p h}^{\alpha}(f)=Q_{p h, 0}(f)+\alpha^{2} Q_{p h, 1}^{\alpha}(f),
$$

where $Q_{p h, 1}^{\alpha}$ is of order 1 when $\alpha$ goes to 0 .

We point out that the extension of the classical Fermi's Golden Rule to the subband case for the description of the electron phonon interaction disregards possible memory effects reported in the literature in the quantum setting $[19,36]$ and considered, e.g., in the non-local collision operator of the quantum SHE model presented in [9]. Here we are accounting for partial quantization of energy induced by the confinement, but the considered time and length scale of the transport phenomena allows for a classical description.

The electron-electron collision operator is given by [34]

$$
\begin{aligned}
= & \sum_{n^{\prime}, r, s} \int_{\left(\mathbb{R}^{2}\right)^{3}}^{Q_{n}(f)_{n}(k)} \Phi_{n, n^{\prime}, r, s}^{e}\left(k, k^{\prime}, k_{1}, k_{1}^{\prime}\right) \delta\left(\varepsilon_{n}+\varepsilon_{n^{\prime}, 1}-\varepsilon_{r}^{\prime}-\varepsilon_{s, 1}^{\prime}\right) \delta\left(k+k_{1}-k^{\prime}-k_{1}^{\prime}\right) \\
& {\left[f_{r}^{\prime} f_{s, 1}^{\prime}\left(1-\eta f_{n}\right)\left(1-\eta f_{n^{\prime}, 1}\right)-f_{n} f_{n^{\prime}, 1}\left(1-\eta f_{r}^{\prime}\right)\left(1-\eta f_{s, 1}^{\prime}\right)\right] d k^{\prime} d k_{1} d k_{1}^{\prime} . }
\end{aligned}
$$

The notation $f_{n^{\prime}, 1}, f_{r}^{\prime}$, and $f_{s, 1}^{\prime}$ stands for $f_{n^{\prime}}\left(k_{1}\right), f_{r}\left(k^{\prime}\right)$, and $f_{s}\left(k_{1}^{\prime}\right)$, respectively.

We define then the elastic collision operator

$$
\begin{aligned}
Q_{0}(f)_{n} & =Q_{i m p}(f)_{n}+Q_{p h, 0}(f)_{n} \\
& =\sum_{n^{\prime} \in \mathbb{N}^{*}} \int_{\mathbb{R}^{2}} \Phi_{n, n^{\prime}}^{0}\left(k, k^{\prime}\right) \delta\left(\varepsilon_{n}(k)-\varepsilon_{n^{\prime}}\left(k^{\prime}\right)\right)\left(f_{n^{\prime}}\left(k^{\prime}\right)-f_{n}(k)\right) d k^{\prime},
\end{aligned}
$$

where $\Phi_{n, n^{\prime}}^{0}=\Phi_{n, n^{\prime}}^{i m p}+\left(2 N_{p h}+1\right) \Phi_{n, n^{\prime}}^{p h}$. We set

$$
Q_{1}^{\alpha}(f)=Q_{p h, 1}^{\alpha}(f)+\frac{1}{\beta} Q_{e}(f) .
$$

Then the kinetic equation, starting point for the diffusive limits, is written in the following form:

$$
\alpha^{2} \partial_{t} f_{n}^{\alpha}+\alpha\left(\nabla_{k} \varepsilon_{n} \cdot \nabla_{x} f_{n}^{\alpha}-\nabla_{x} \varepsilon_{n} \cdot \nabla_{k} f_{n}^{\alpha}\right)=Q_{0}\left(f^{\alpha}\right)_{n}+\alpha^{2} Q_{1}^{\alpha}\left(f^{\alpha}\right)_{n} .
$$

2.2. Definitions and notations. We first recall the coarea formula: for any $C^{1}$ function $g: B \mapsto \mathbb{R}$, and any test function $\psi \in C^{0}(B)$, we have

$$
\int_{B} \psi(k) d k=\int_{\mathbb{R}}\left(\int_{g^{-1}(\varepsilon)} \psi(k) \frac{d S_{\varepsilon}(k)}{|\nabla g(k)|}\right) d \varepsilon,
$$

where $d S_{\varepsilon}(k)$ denotes the Euclidean surface element on the manifold $g^{-1}(\varepsilon)$. We denote $d N_{\varepsilon}(k)=d S_{\varepsilon}(k) /|\nabla g(k)|$. Taking $g(k)=|k|^{2} \hbar^{2} /\left(2 m^{*}\right)$, the set of possible wave vectors of electrons belonging to the $n$-th subband and having total energy $\varepsilon$ is given by $S_{\varepsilon-\epsilon_{n}}=\left\{k \in \mathbb{R}^{2}\right.$ s.t. $\left.|k|^{2}=2 m^{*} \hbar^{-2}\left(\varepsilon-\epsilon_{n}\right)\right\}$ and $d N_{\varepsilon-\epsilon_{n}}=\frac{d S_{\varepsilon-\epsilon_{n}}}{|k| \hbar^{2} / m^{*}}$, where $d S_{\varepsilon-\epsilon_{n}}$ is the surface measure of the ball $S_{\varepsilon-\epsilon_{n}}$. The coarea formula leads to

$$
\sum_{n \in \mathbb{N}^{*}} \int_{\mathbb{R}^{2}} \psi_{n}(k) d k=\sum_{n \in \mathbb{N}^{*}} \int_{\epsilon_{n}}^{+\infty}\left(\int_{S_{\varepsilon-\epsilon_{n}}} \psi_{n}(k) d N_{\varepsilon-\epsilon_{n}}(k)\right) d \varepsilon
$$


and

$$
\sum_{n \in \mathbb{N}^{*}} \int_{\mathbb{R}^{2}} \psi_{n}(k) \delta\left(\epsilon_{n}+\frac{|k|^{2} \hbar^{2}}{2 m^{*}}-\varepsilon\right) d k=\sum_{n \in \mathbb{N}^{*}} \int_{S_{\varepsilon-\epsilon_{n}}} \psi_{n}(k) d N_{\varepsilon-\epsilon_{n}}(k) .
$$

DEFINITION 2.1. We will use the following notations:

- The density of states is defined by

$$
N(t, x, \varepsilon):=\sum_{n \in \mathbb{N}^{*}} \int_{S_{\varepsilon-\epsilon_{n}}} d N_{\varepsilon-\epsilon_{n}}(k)=2 \pi \frac{m^{*}}{\hbar^{2}} \mathcal{N}(t, x, \varepsilon),
$$

where $\mathcal{N}(t, x, \varepsilon)=\max \left\{n \in \mathbb{N}^{*} \quad / \epsilon_{n}(t, x) \leq \varepsilon\right\}$, with the convention of $\mathcal{N}(t, x, \varepsilon)=0$ if $\varepsilon<\epsilon_{1}(t, x)$.

- The Fermi-Dirac function is given by

$$
\mathcal{F}_{\mu, T}(t, x, \varepsilon)=\left(\eta+\exp \left(\frac{\varepsilon-\mu}{k_{B} T}\right)\right)^{-1} .
$$

- We introduce the following two Hilbert spaces:

$$
\mathbb{L}^{2}:=\left\{f=\left(f_{n}\right)_{n \in \mathbb{N}^{*}}, \quad \sum_{n=1}^{+\infty} \int_{\mathbb{R}^{2}}\left|f_{n}(k)\right|^{2} d k<+\infty\right\},
$$

endowed with the natural scalar product

$$
\langle f, g\rangle=\sum_{n \in \mathbb{N}^{*}} \int_{\mathbb{R}^{2}} f_{n}(k) g_{n}(k) d k
$$

and

$$
\mathbb{L}_{\mathcal{F}}^{2}=\left\{f \in L^{2}(\mathbb{R}) \text { s.t. } \int_{\mathbb{R}} f^{2}(\varepsilon) \frac{d \varepsilon}{\mathcal{F}(\varepsilon)(1-\eta \mathcal{F}(\varepsilon))}<+\infty\right\},
$$

endowed with the weighted scalar product defined by

$$
\langle f, g\rangle_{\mathcal{F}}=\int_{\mathbb{R}} f(\varepsilon) g(\varepsilon) \frac{d \varepsilon}{\mathcal{F}(\varepsilon)(1-\eta \mathcal{F}(\varepsilon))} .
$$

We will make the following assumption on the cross-section.

Assumption 2.2. The coefficient $\Phi_{n, n^{\prime}}^{0}$ satisfies, for $\lambda_{0}$ and $\lambda_{1}$ two positive constants,

$$
0<\lambda_{0}<\Phi_{n, n^{\prime}}^{0} N\left(t, x, \varepsilon_{n}\right)<\lambda_{1}, \quad \Phi_{n, n^{\prime}}^{0}\left(k, k^{\prime}\right)=\Phi_{n^{\prime}, n}^{0}\left(k^{\prime}, k\right),
$$

where $N$ is the density of state defined above.

2.3. First macroscopic scaling: The spherical harmonic expansion model. For the sake of completeness of this work, we present in this section the limit $\alpha \rightarrow 0$ of the kinetic equation (2.9). All calculations will be done formally and we refer the reader to [7], where the rigorous derivation is studied. We consider the Hilbert expansion

$$
f^{\alpha}=f^{0}+\alpha f^{1}+\alpha^{2} f^{2}+\cdots .
$$


By linearity of the operator $Q_{0}$ and by identifying the terms of equal powers of $\alpha$ in (2.9), we obtain

$$
\begin{aligned}
& Q_{0}\left(f^{0}\right)=0 \\
& Q_{0}\left(f^{1}\right)_{n}=\frac{\hbar^{2} k}{m^{*}} \cdot \nabla_{x} f_{n}^{0}-\nabla_{x} \epsilon_{n} \cdot \nabla_{k} f_{n}^{0}, \\
& Q_{0}\left(f^{2}\right)_{n}=\partial_{t} f_{n}^{0}+\frac{\hbar^{2} k}{m^{*}} \cdot \nabla_{x} f_{n}^{1}-\nabla_{x} \epsilon_{n} \cdot \nabla_{k} f_{n}^{1}-Q_{1}^{0}\left(f^{0}\right)_{n},
\end{aligned}
$$

where $Q_{1}^{0}$ is obtained by taking $\alpha=0$ in the expression (2.8).

We will then make use of the following properties of the collision operator (see $[7])$.

Proposition 2.3. Under Assumption 2.2, the elastic collision operator $Q_{0}$ defined in (2.7) satisfies the following properties:

1. The linear operator $Q_{0}: \mathbb{L}^{2} \mapsto \mathbb{L}^{2}$ is a bounded, symmetric, non-positive operator.

2. For any bounded function $\psi: \mathbb{R} \mapsto \mathbb{R}$, we denote $\psi(\varepsilon)_{n}(k)=\psi\left(\frac{|k|^{2} \hbar^{2}}{2 m^{*}}+\epsilon_{n}\right)$. Then,

$$
\forall f \in \mathbb{L}^{2}, Q_{0}(\psi(\varepsilon) f)=\psi(\varepsilon) Q_{0}(f) .
$$

3. The kernel of $Q_{0}$ is the set

$$
\operatorname{Ker} Q_{0}=\left\{f \in \mathbb{L}^{2}, \quad \text { s.t. } \exists \psi: \mathbb{R} \rightarrow \mathbb{R}, \quad f=\psi(\varepsilon)\right\} .
$$

4. The range $R\left(Q_{0}\right)$ is closed and coincides with the orthogonal complement of the kernel of $Q_{0}$ given by

$$
\left(\operatorname{Ker} Q_{0}\right)^{\perp}=\left\{f \in \mathbb{L}^{2}, \quad \text { s.t. } \sum_{n \in \mathbb{N}^{*}} \int_{S_{\varepsilon-\epsilon_{n}}} f_{n}(k) d N_{\varepsilon-\epsilon_{n}}(k)=0 \text {, for a.e. } \varepsilon \geq \epsilon_{1}\right\} \text {. }
$$
tion:

From Proposition 2.3 and (2.11), we deduce that $f^{0}$ is an energy dependent func-

$$
f_{n}^{0}(t, x, k)=F\left(t, x, \varepsilon_{n}\right) .
$$

Choosing $\psi: \mathbb{R} \rightarrow \mathbb{R}$ such that $k \psi(\varepsilon) \in \mathbb{L}^{2}$, we deduce from Proposition 2.3 that there exists a unique solution $\xi=\left(\xi_{1}, \xi_{2}\right)$ with $\xi_{i}$ in $\left(\operatorname{Ker} Q_{0}\right)^{\perp}$ such that

$$
-Q_{0}\left(\xi_{i}\right)=\frac{\hbar^{2} k_{i}}{m^{*}} \psi(\varepsilon), \quad i=1,2 .
$$

We introduce $\Theta$ such that $\xi=\Theta \psi(\varepsilon)$. From the second item of Proposition 2.3, $\Theta$ is independent of the choice of the function $\psi$. Then, the unique solution in $\left(\operatorname{Ker} Q_{0}\right)^{\perp}$ of equation (2.12) is given by

$$
f_{n}^{1}(t, x, k)=-\Theta_{n}(t, x, k) \cdot \nabla_{x} F\left(t, x, \varepsilon_{n}\right) .
$$

Finally, the solvability condition of equation (2.13) is that the right hand side belongs to $\left(\operatorname{Ker} Q_{0}\right)^{\perp}$. This leads to

$$
\sum_{n \in \mathbb{N}^{*}} \int_{S_{\varepsilon-\epsilon_{n}}}\left(\partial_{t} f_{n}^{0}+\nabla_{k} \varepsilon_{n} \cdot \nabla_{x} f_{n}^{1}-\nabla_{x} \epsilon_{n} \cdot \nabla_{k} f_{n}^{1}-Q_{1}^{0}\left(f^{0}\right)_{n}\right) d N_{\varepsilon-\epsilon_{n}}(k)=0,
$$


for a.e. $\varepsilon \geq \epsilon_{1}$. Let us denote

$S_{e}(F)=\sum_{n \in \mathbb{N}^{*}} \int_{S_{\varepsilon-\epsilon_{n}}} Q_{e}(F)_{n} d N_{\varepsilon-\epsilon_{n}}(k)$, and $S_{1}(F)=\sum_{n \in \mathbb{N}^{*}} \int_{S_{\varepsilon-\epsilon_{n}}} Q_{p h, 1}^{0}(F)_{n} d N_{\varepsilon-\epsilon_{n}}(k)$.

Multiplying (2.15) by an energy-dependent test function $\phi(\varepsilon)$ and integrating with respect to the variable $\varepsilon$, we obtain for the first term

$$
\begin{aligned}
& \int_{\epsilon_{1}}^{\infty} \sum_{n \in \mathbb{N}^{*}} \int_{S_{\varepsilon-\epsilon_{n}}} \partial_{t} f_{n}^{0} d N_{\varepsilon-\epsilon_{n}}(k) \phi(\varepsilon) d \varepsilon \\
= & \int_{\epsilon_{1}}^{\infty} \sum_{n \in \mathbb{N}^{*}} \int_{S_{\varepsilon-\epsilon_{n}}}\left(\partial_{t} F+\partial_{\varepsilon} F \partial_{t} \epsilon_{n}\right) d N_{\varepsilon-\epsilon_{n}}(k) \phi(\varepsilon) d \varepsilon \\
= & \int_{\epsilon_{1}}^{\infty} N \partial_{t} F \phi(\varepsilon) d \varepsilon+\int_{\epsilon_{1}}^{\infty} \partial_{\varepsilon} F\left(\sum_{n \in \mathbb{N}^{*}} \partial_{t} \epsilon_{n} \int_{S_{\varepsilon-\epsilon_{n}}} d N_{\varepsilon-\epsilon_{n}}(k)\right) \phi(\varepsilon) d \varepsilon .
\end{aligned}
$$

Using the coarea formula (2.10), we deduce that

$$
\begin{aligned}
& \int_{\epsilon_{1}}^{\infty}\left(\sum_{n \in \mathbb{N}^{*}} \int_{S_{\varepsilon-\epsilon_{n}}}\left(\nabla_{k} \varepsilon_{n} \cdot \nabla_{x} f_{n}^{1}-\nabla_{x} \epsilon_{n} \cdot \nabla_{k} f_{n}^{1}\right) d N_{\varepsilon-\epsilon_{n}}(k)\right) \phi(\varepsilon) d \varepsilon \\
= & \sum_{n \in \mathbb{N}^{*}} \int_{\mathbb{R}^{2}}\left(\nabla_{x} \cdot\left(\frac{\hbar^{2} k}{m^{*}} f_{n}^{1}\right)-\nabla_{k} \cdot\left(f_{n}^{1} \nabla_{x} \epsilon_{n}\right)\right) \phi\left(\varepsilon_{n}\right) d k \\
= & \nabla_{x} \cdot\left(\sum_{n \in \mathbb{N}^{*}} \int_{\mathbb{R}^{2}} \frac{\hbar^{2} k}{m^{*}} f_{n}^{1} \phi\left(\varepsilon_{n}\right) d k\right) \\
= & -\int_{\epsilon_{1}}^{\infty} \nabla_{x} \cdot\left(\sum_{n \in \mathbb{N}^{*}} \int_{S_{\varepsilon-\epsilon_{n}}} \frac{\hbar^{2} k}{m^{*}} \otimes \Theta_{n} d N_{\varepsilon-\epsilon_{n}}(k) \cdot \nabla_{x} F\right) \phi(\varepsilon) d \varepsilon,
\end{aligned}
$$

where the last identity is a consequence of (2.14). We define the diffusion matrix by

$$
D(t, x, \varepsilon):=\sum_{n \in \mathbb{N}^{*}} \int_{S_{\varepsilon-\epsilon_{n}}} \frac{\hbar^{2} k}{m^{*}} \otimes \Theta_{n} d N_{\varepsilon-\epsilon_{n}}(k),
$$

and the current density by

$$
J(t, x, \varepsilon)=-D(t, x, \varepsilon) \cdot \nabla_{x} F(t, x, \varepsilon) .
$$

With these notations, we get that in the distributional sense, equation (2.15) is equivalent to the spherical harmonic expansion (SHE) model

$$
N \partial_{t} F+\nabla_{x} \cdot J-\kappa \partial_{\varepsilon} F=\frac{1}{\beta} S_{e}(F)+S_{1}(F),
$$

where $\kappa$ is given by

$$
\kappa(t, x, \varepsilon)=-2 \pi \frac{m^{*}}{\hbar^{2}} \partial_{t}\left(\sum_{n \in \mathbb{N}^{*}}\left(\varepsilon-\epsilon_{n}\right)^{+}\right) .
$$

The notation $u^{+}=\max \{0, u\}$ denotes the positive part of $u$. We recall moreover a property of the diffusion matrix $D$ stated in Lemma 2.8 of [7]. We point out that the effect of the confinement is reflected in the special form of the coefficients of (2.19), which involve the subband energies. 
Lemma 2.4. The diffusion matrix $D(t, x, \varepsilon)$ defined in $(2.17)$ is a symmetric and nonnegative $2 \times 2$ matrix.

REMARK 2.5. We end this section with a particular choice of the cross-section $\Phi_{n, n^{\prime}}^{0}$, which allows us to explicitly compute the diffusion matrix. If the cross-section is an energy-dependent function, which, due to $(2.7)$, needs only to be defined on the set $\left\{\varepsilon_{n^{\prime}}\left(k^{\prime}\right)=\varepsilon_{n}(k)\right\}$ and which is given by

$$
\Phi_{n, n^{\prime}}^{0}\left(t, x, k, k^{\prime}\right)=\Phi^{0}\left(t, x, \varepsilon_{n}\right),
$$

then, after a straightforward computation, we have that

$$
\Theta_{n}(t, x, k)=\frac{1}{\Phi^{0}\left(t, x, \varepsilon_{n}\right) N\left(t, x, \varepsilon_{n}\right)} \nabla_{k} \varepsilon_{n} .
$$

Therefore, the diffusion matrix defined in (2.17) has the expression

$$
D(t, x, \varepsilon)=\frac{\pi \hbar^{2} / m^{*}}{\Phi^{0}(t, x, \varepsilon) N(t, x, \varepsilon)} \sum_{n \in \mathbb{N}^{*}}\left(\varepsilon-\epsilon_{n}\right)^{+} I d .
$$

2.4. Second macroscopic scaling: The energy-transport model. We start from the SHE model (2.19) and we assume that the electron-electron collision operator is dominant with respect to the second order correction of the phonon collision operator and therefore $\beta \ll 1$, in order to obtain an ET model. Passing through the SHE model, instead of starting directly from the Boltzmann equation, allows one to get an explicit expression of the coefficients, which is needed for numerical purposes. Moreover, the considered dominant scattering mechanisms provide an energy relaxation term in the macroscopic limiting model.

The formal limit $\beta \rightarrow 0$ in (2.19) is again performed by means of a Hilbert expansion

$$
F=F^{0}+\beta F^{1}+\cdots .
$$

Identifying equal powers of $\beta$ implies

$$
\begin{gathered}
S_{e}\left(F_{0}\right)=0 \\
N \partial_{t} F^{0}+\nabla_{x} J^{0}-\kappa \partial_{\varepsilon} F^{0}-S_{1}\left(F^{0}\right)=D_{F^{0}} S_{e}\left(F^{1}\right),
\end{gathered}
$$

where $D_{F^{0}} S_{e}$ denotes the Fréchet derivative of $S_{e}$ at $F^{0}$.

We summarize below some useful properties of the collision operator $S_{e}$ defined in (2.16) and of its Fréchet derivative.

Proposition 2.6. Under micro-reversibility assumptions on the cross-section $\Phi^{e}$, the operator $S_{e}$ satisfies the following properties:

(i) For all $f, g \in L^{2}(\mathbb{R})$, we have

$$
\begin{aligned}
& \int_{\mathbb{R}} S_{e}(f)(\varepsilon) g(\varepsilon) d \varepsilon \\
= & -\frac{1}{4} \sum_{n, n^{\prime}, r, s} \int_{\left(\mathbb{R}^{2}\right)^{4}} \Phi_{n, n^{\prime}, r, s}^{e} \delta_{\varepsilon} \delta_{k}\left[f\left(\varepsilon_{r}\left(k^{\prime}\right)\right) f\left(\varepsilon_{s}\left(k_{1}^{\prime}\right)\right)\left(1-\eta f\left(\varepsilon_{n}(k)\right)\right)\left(1-\eta f\left(\varepsilon_{n^{\prime}}\left(k_{1}\right)\right)\right)\right.
\end{aligned}
$$


108

ENERGY-TRANSPORT SUBBAND MODEL

$$
\begin{aligned}
& \left.-f\left(\varepsilon_{n}(k)\right) f\left(\varepsilon_{n^{\prime}}\left(k_{1}\right)\right)\left(1-\eta f\left(\varepsilon_{r}\left(k^{\prime}\right)\right)\right)\left(1-\eta f\left(\varepsilon_{s}\left(k_{1}^{\prime}\right)\right)\right)\right] \\
& {\left[g_{r}^{\prime}+g_{s, 1}^{\prime}-g_{n}-g_{n^{\prime}, 1}\right] d k d k_{1} d k^{\prime} d k_{1}^{\prime} .}
\end{aligned}
$$

(ii) Its kernel is given by

$$
\operatorname{Ker} S_{e}=\left\{f \in L^{2}(\mathbb{R}) ; \exists \mu(t, x), T(t, x) \text { s.t. } f(t, x, \varepsilon)=\mathcal{F}_{\mu, T}(t, x, \varepsilon)\right\},
$$

where $\mathcal{F}_{\mu, T}$ is the so-called Fermi-Dirac distribution function (see Definition 2.1).

Proposition 2.7. The linear operator $D_{\mathcal{F}} S_{e}$ satisfies

(i) $D_{\mathcal{F}} S_{e}$ is bounded, symmetric, non-positive on $\mathbb{L}_{\mathcal{F}}^{2}$.

(ii) The kernel of $D_{\mathcal{F}} S_{e}$ is given by

$$
\operatorname{Ker}\left(D_{\mathcal{F}} S_{e}\right)=\operatorname{Span}\{\mathcal{F}(1-\eta \mathcal{F}), \mathcal{F}(1-\eta \mathcal{F}) \varepsilon\} .
$$

(iii) The range of $D_{\mathcal{F}} S_{e}$ is closed and we have

$$
R\left(D_{\mathcal{F}} S_{e}\right)=\operatorname{Ker}\left(D_{\mathcal{F}} S_{e}\right)^{\perp}=\left\{f \in \mathbb{L}_{\mathcal{F}}^{2} \quad ; \quad \int_{\mathbb{R}} f(\varepsilon)\left(\begin{array}{l}
1 \\
\varepsilon
\end{array}\right) d \varepsilon=0\right\} .
$$

These properties are an easy consequence of Proposition 3.16, Proposition 3.17, and Proposition 3.19 of [7], using the fact that

$$
\begin{aligned}
\int_{\mathbb{R}} S_{e}(f)(\varepsilon) g(\varepsilon) d \varepsilon & =\int_{\epsilon_{1}}^{+\infty} \sum_{n \in \mathbb{N}^{*}} \int_{S_{\varepsilon-\epsilon_{n}}} Q_{e}(f)_{n}(\varepsilon) g(\varepsilon) d N_{\varepsilon-\epsilon_{n}}(k) d \varepsilon \\
& =\sum_{n \in \mathbb{N}^{*}} \int_{\epsilon_{n}}^{+\infty} \int_{S_{\varepsilon-\epsilon_{n}}} Q_{e}(f)_{n}(\varepsilon) g(\varepsilon) d N_{\varepsilon-\epsilon_{n}}(k) d \varepsilon \\
& =\sum_{n \in \mathbb{N}^{*}} \int_{\mathbb{R}^{2}} Q_{e}(f)_{n}\left(\varepsilon_{n}\right) g\left(\varepsilon_{n}\right) d k
\end{aligned}
$$

where we use the coarea formula (2.10) for the last identity.

Formal derivation of energy-transport model. Let us come back to the formal limit $\beta \rightarrow 0$ in (2.19). Thanks to Proposition 2.6, equation (2.22) implies that there exist $\mu(t, x)$ and $T(t, x)$ such that

$$
F^{0}(t, x, \varepsilon)=\mathcal{F}_{\mu, T}(t, x, \varepsilon)
$$

From Proposition 2.7 we deduce that equation (2.23) admits a solution if and only if

$$
\int_{\mathbb{R}}\left(N \partial_{t} F^{0}+\nabla_{x} \cdot J^{0}-\kappa \partial_{\varepsilon} F^{0}-S_{1}\left(F^{0}\right)\right)\left(\begin{array}{l}
1 \\
\varepsilon
\end{array}\right) d \varepsilon=0 .
$$

For the first term, the definition of the density of states $N$ (see Definition 2.1) implies that $N(t, x, \varepsilon)=2 \pi \frac{m^{*}}{\hbar^{2}} n$ if $\varepsilon \in\left[\epsilon_{n}, \epsilon_{n+1}\right)$, and vanishes for $\varepsilon<\epsilon_{1}$. Then

$$
\begin{aligned}
\int_{\mathbb{R}} N \partial_{t} F^{0}\left(\begin{array}{l}
1 \\
\varepsilon
\end{array}\right) d \varepsilon & =\sum_{n \in \mathbb{N}^{*}} \int_{\epsilon_{n}}^{\epsilon_{n+1}} 2 \pi \frac{m^{*}}{\hbar^{2}} n \partial_{t} F_{0}\left(\begin{array}{l}
1 \\
\varepsilon
\end{array}\right) d \varepsilon \\
& =\partial_{t}\left(\int_{\mathbb{R}} N F^{0}\left(\begin{array}{l}
1 \\
\varepsilon
\end{array}\right) d \varepsilon\right)-2 \pi \frac{m^{*}}{\hbar^{2}} \sum_{n \in \mathbb{N}^{*}} \partial_{t} \epsilon_{n} F^{0}\left(\epsilon_{n}\right)\left(\begin{array}{c}
1 \\
\epsilon_{n}
\end{array}\right) .
\end{aligned}
$$


Using the expression of the current (2.18), we can rewrite the second term of (2.25):

$$
\int_{\mathbb{R}} \nabla_{x} \cdot J^{0}\left(\begin{array}{l}
1 \\
\varepsilon
\end{array}\right) d \varepsilon=-\nabla_{x} \cdot\left[\int_{\mathbb{R}} D(t, x, \varepsilon) \cdot \nabla_{x} F^{0}(t, x, \varepsilon)\left(\begin{array}{l}
1 \\
\varepsilon
\end{array}\right) d \varepsilon\right] .
$$

From (2.20), we deduce

$$
\begin{aligned}
& \int_{\mathbb{R}} \kappa \partial_{\varepsilon} F^{0}\left(\begin{array}{l}
1 \\
\varepsilon
\end{array}\right) d \varepsilon \\
= & \sum_{n \in \mathbb{N}^{*}} \int_{\epsilon_{n}}^{\infty} 2 \pi \frac{m^{*}}{\hbar^{2}} \partial_{t} \epsilon_{n} \partial_{\varepsilon} F^{0}\left(\begin{array}{c}
1 \\
\varepsilon
\end{array}\right) d \varepsilon \\
= & -\sum_{n \in \mathbb{N}^{*}} 2 \pi \frac{m^{*}}{\hbar^{2}} \partial_{t} \epsilon_{n} F^{0}\left(\epsilon_{n}\right)\left(\begin{array}{c}
1 \\
\epsilon_{n}
\end{array}\right)-\sum_{n \in \mathbb{N}^{*}} 2 \pi \frac{m^{*}}{\hbar^{2}} \partial_{t} \epsilon_{n} \int_{\epsilon_{n}}^{\infty} F^{0}(\varepsilon)\left(\begin{array}{c}
0 \\
1
\end{array}\right) d \varepsilon,
\end{aligned}
$$

where we use an integration by parts for the last identity. Finally, the solvability condition (2.25) writes in the following form:

$$
\begin{aligned}
\partial_{t}\left(\int_{\mathbb{R}} N F^{0}\left(\begin{array}{l}
1 \\
\varepsilon
\end{array}\right) d \varepsilon\right) & +\sum_{n \in \mathbb{N}^{*}} 2 \pi \frac{m^{*}}{\hbar^{2}} \partial_{t} \epsilon_{n} \int_{\epsilon_{n}}^{\infty} F^{0}(\varepsilon)\left(\begin{array}{l}
0 \\
1
\end{array}\right) d \varepsilon \\
& -\nabla_{x} \cdot\left[\int_{\mathbb{R}} D(t, x, \varepsilon) \cdot \nabla_{x} F^{0}(t, x, \varepsilon)\left(\begin{array}{l}
1 \\
\varepsilon
\end{array}\right) d \varepsilon\right]=\int_{\mathbb{R}} S_{1}\left(F^{0}\right)\left(\begin{array}{l}
1 \\
\varepsilon
\end{array}\right) d \varepsilon .
\end{aligned}
$$

Let us denote by $\rho$ and $\rho \mathcal{E}$ the charge density and the energy density, respectively, associated to the Fermi-Dirac distribution function $\mathcal{F}_{\mu, T}$ :

$$
\begin{array}{r}
\rho_{\mu, T}(t, x):=\int_{\mathbb{R}} N \mathcal{F}_{\mu, T}(t, x, \varepsilon) d \varepsilon=\sum_{n \in \mathbb{N}^{*}} \int_{\mathbb{R}^{2}} \mathcal{F}_{\mu, T}\left(t, x, \varepsilon_{n}\right) d k, \\
\rho \mathcal{E}_{\mu, T}(t, x):=\int_{\mathbb{R}} N \mathcal{F}_{\mu, T}(t, x, \varepsilon) \varepsilon d \varepsilon=\sum_{n \in \mathbb{N}^{*}} \int_{\mathbb{R}^{2}} \varepsilon_{n} \mathcal{F}_{\mu, T}\left(t, x, \varepsilon_{n}\right) d k .
\end{array}
$$

We can state easily that for a Fermi-Dirac function, we have

$$
\nabla_{x} \mathcal{F}_{\mu, T}(t, x, \varepsilon)=-\mathcal{F}_{\mu, T}\left(1-\eta \mathcal{F}_{\mu, T}\right)\left(\varepsilon \nabla_{x}\left(\frac{1}{k_{B} T}\right)-\nabla_{x}\left(\frac{\mu}{k_{B} T}\right)\right) .
$$

Then equation (2.26) reads, using $\int_{\mathbb{R}} S_{1}(\mathcal{F}) d \varepsilon=0$,

$$
\begin{gathered}
\partial_{t} \rho_{\mu, T}-\nabla_{x} \cdot J_{1}=0 \\
\partial_{t}\left(\rho \mathcal{E}_{\mu, T}\right)+\sum_{n \in \mathbb{N}^{*}} 2 \pi \frac{m^{*}}{\hbar^{2}} \partial_{t} \epsilon_{n} \int_{\epsilon_{n}}^{\infty} \mathcal{F}_{\mu, T}(t, x, \varepsilon) d \varepsilon-\nabla_{x} \cdot J_{2}=W
\end{gathered}
$$

where we denote

$$
\begin{aligned}
& J_{1}:=\int_{\mathbb{R}} D(t, x, \varepsilon) \cdot \nabla_{x} \mathcal{F}_{\mu, T}(t, x, \varepsilon) d \varepsilon=\mathbb{D}_{00} \nabla_{x}\left(\frac{\mu}{k_{B} T}\right)-\mathbb{D}_{01} \nabla_{x}\left(\frac{1}{k_{B} T}\right), \\
& J_{2}:=\int_{\mathbb{R}} \varepsilon D(t, x, \varepsilon) \cdot \nabla_{x} \mathcal{F}_{\mu, T}(t, x, \varepsilon) d \varepsilon=\mathbb{D}_{10} \nabla_{x}\left(\frac{\mu}{k_{B} T}\right)-\mathbb{D}_{11} \nabla_{x}\left(\frac{1}{k_{B} T}\right),
\end{aligned}
$$


and where the diffusion coefficients are defined by

$$
\mathbb{D}_{i j}(t, x)=\int_{\mathbb{R}} D(t, x, \varepsilon) \varepsilon^{i+j} \mathcal{F}_{\mu, T}\left(1-\eta \mathcal{F}_{\mu, T}\right) d \varepsilon, \quad \text { for } \quad i, j=0,1,
$$

with $D$ being defined in (2.17). The system (2.29)-(2.33) forms the energy-transport model in the transport direction for a partially confined electron gas. We recover the general form for energy-transport models for semiconductors (see [3, 4, 25] and references therein). The right hand side of the energy equation (2.30) is the so-called relaxation term $W$ defined by

$$
W:=\int_{\mathbb{R}} \varepsilon S_{1}\left(\mathcal{F}_{\mu, T}\right) d \varepsilon=\sum_{n \in \mathbb{N}^{*}} \int_{\epsilon_{n}}^{+\infty}\left(\int_{S_{\varepsilon-\epsilon_{n}}} Q_{p h, 1}^{0}(f)_{n} d N_{\varepsilon-\epsilon_{n}}(k)\right) \varepsilon d \varepsilon .
$$

As for the SHE model (2.19), the presence of the subband energies in the diffusion matrix and in the relaxation term reflects the effect of the confinement in the transport equation.

Then we have the following important property for the diffusion matrix which is an easy consequence of expression (2.33) and Lemma 2.4.

Lemma 2.8. The diffusion matrix

$$
\mathcal{D}:=\left(\begin{array}{ll}
\mathbb{D}_{00} & \mathbb{D}_{01} \\
\mathbb{D}_{10} & \mathbb{D}_{11}
\end{array}\right)
$$

is symmetric and positive definite.

REMARK 2.9. After straightforward calculations, we can have an explicit expression of $\rho$ and $\rho \mathcal{E}$. In fact,

$$
\begin{array}{r}
\rho_{\mu, T}(t, x)=\sum_{n \in \mathbb{N}^{*}} \int_{\epsilon_{n}}^{\epsilon_{n+1}} 2 \pi \frac{m^{*}}{\hbar^{2}} n \mathcal{F}_{\mu, T}(t, x, \varepsilon) d \varepsilon \\
=\frac{2 \pi m^{*} k_{B} T}{\eta \hbar^{2}} \sum_{n \in \mathbb{N}^{*}} \log \left(1+\eta \exp \left(\frac{\mu-\epsilon_{n}}{k_{B} T}\right)\right), \\
\rho \mathcal{E}_{\mu, T}(t, x)=\sum_{n \in \mathbb{N}^{*}} 2 \pi \frac{m^{*}}{\hbar^{2}} n \int_{\epsilon_{n}}^{\epsilon_{n+1}} \varepsilon \mathcal{F}_{\mu, T}(t, x, \varepsilon) d \varepsilon,
\end{array}
$$

if $\eta>0$. For Boltzmann statistics $\eta=0$, we have

$$
\begin{gathered}
\rho_{\mu, T}(t, x)=\frac{2 \pi m^{*} k_{B} T(t, x)}{\hbar^{2}} \sum_{n \in \mathbb{N}^{*}} \exp \left(\frac{\mu(t, x)-\epsilon_{n}(t, x)}{k_{B} T(t, x)}\right), \\
\rho \mathcal{E}_{\mu, T}(t, x)=\sum_{n \in \mathbb{N}^{*}} 2 \pi \frac{m^{*}}{\hbar^{2}} k_{B} T(t, x)\left(\epsilon_{n}(t, x)+T(t, x)\right) \exp \left(\frac{\mu(t, x)-\epsilon_{n}(t, x)}{k_{B} T(t, x)}\right) .
\end{gathered}
$$


2.5. Relaxation term W. The relaxation term defined in (2.34) measures the influence of the interaction of phonons with the charge carriers. In [32] we have formally established that a diffusion limit of the kinetic Boltzmann transport equation coupled to the subband model in the scaling of dominant phonon-electron interaction leads to a drift-diffusion system in the transport direction coupled to the subband model. Using the coarea formula we can rewrite (2.34) as

$$
W=\sum_{n \in \mathbb{N}^{*}} \int_{\mathbb{R}^{2}} \varepsilon_{n} Q_{p h, 1}^{0}\left(\mathcal{F}_{\mu, T}\right) d k
$$

Moreover, we have

$$
\begin{aligned}
& \sum_{n \in \mathbb{N}^{*}} \int_{\mathbb{R}^{2}} \varepsilon_{n} Q_{p h}^{\alpha}\left(\mathcal{F}_{\mu, T}\right)_{n} d k \\
& =\sum_{n, n^{\prime}} \int_{\mathbb{R}^{4}} \Phi_{n, n^{\prime}}^{p h}\left(k, k^{\prime}\right)\left(\left[\left(N_{p h}+1\right) \delta\left(\varepsilon_{n}-\varepsilon_{n^{\prime}}^{\prime}+\alpha^{2} \varepsilon_{p h}\right)\right.\right. \\
& \left.\quad+N_{p h} \delta\left(\varepsilon_{n}-\varepsilon_{n^{\prime}}^{\prime}-\alpha^{2} \varepsilon_{p h}\right)\right] \varepsilon_{n} \mathcal{F}_{n^{\prime}}^{\prime}\left(1-\eta \mathcal{F}_{n}\right) \\
& \left.\quad-\left[\left(N_{p h}+1\right) \delta\left(\varepsilon_{n^{\prime}}^{\prime}-\varepsilon_{n}+\alpha^{2} \varepsilon_{p h}\right)+N_{p h} \delta\left(\varepsilon_{n^{\prime}}^{\prime}-\varepsilon_{n}-\alpha^{2} \varepsilon_{p h}\right)\right] \varepsilon_{n} \mathcal{F}_{n}\left(1-\eta \mathcal{F}_{n^{\prime}}^{\prime}\right)\right) d k d k^{\prime}
\end{aligned}
$$

where the notation $\varepsilon_{n^{\prime}}^{\prime}, \mathcal{F}_{n}$, and $\mathcal{F}_{n^{\prime}}^{\prime}$ stands, respectively, for $\varepsilon_{n^{\prime}}\left(k^{\prime}\right), \mathcal{F}_{\mu, T}\left(\varepsilon_{n}\right)$, and $\mathcal{F}_{\mu, T}\left(\varepsilon_{n^{\prime}}^{\prime}\right)$. Since the Fermi-Dirac distribution function is energy-dependent, we have

$$
\begin{aligned}
& \sum_{n \in \mathbb{N}^{*}} \int_{\mathbb{R}^{2}} \varepsilon_{n} Q_{p h}^{\alpha}\left(\mathcal{F}_{\mu, T}\right)_{n} d k \\
& =\sum_{n, n^{\prime}} \int_{\mathbb{R}^{4}} \Phi_{n, n^{\prime}}^{p h}\left(k, k^{\prime}\right)\left(1-\eta \mathcal{F}_{n^{\prime}}^{\prime}\right)\left(1-\eta \mathcal{F}_{n}\right)\left(\left[\left(N_{p h}+1\right) \delta\left(\varepsilon_{n}-\varepsilon_{n^{\prime}}^{\prime}+\alpha^{2} \varepsilon_{p h}\right)\right.\right. \\
& \left.\quad+N_{p h} \delta\left(\varepsilon_{n}-\varepsilon_{n^{\prime}}^{\prime}-\alpha^{2} \varepsilon_{p h}\right)\right] \varepsilon_{n} M_{n^{\prime}}^{\prime} \\
& \left.\quad-\left[\left(N_{p h}+1\right) \delta\left(\varepsilon_{n^{\prime}}^{\prime}-\varepsilon_{n}+\alpha^{2} \varepsilon_{p h}\right)+N_{p h} \delta\left(\varepsilon_{n^{\prime}}^{\prime}-\varepsilon_{n}-\alpha^{2} \varepsilon_{p h}\right)\right] \varepsilon_{n} M_{n}\right) d k d k^{\prime},
\end{aligned}
$$

where $M_{n}=e^{\left(\mu-\varepsilon_{n}\right) /\left(k_{B} T\right)}$ is the Maxwellian and it satisfies $\mathcal{F}_{n}=M_{n}\left(1-\eta \mathcal{F}_{n}\right)$. Moreover,

$$
\begin{aligned}
& \sum_{n \in \mathbb{N}^{*}} \int_{\mathbb{R}^{2}} \varepsilon_{n} Q_{p h}^{\alpha}\left(\mathcal{F}_{\mu, T}\right)_{n} d k \\
= & \sum_{n, n^{\prime}} \int_{\mathbb{R}^{4}} \Phi_{n, n^{\prime}}^{p h}\left(k, k^{\prime}\right)\left(1-\eta \mathcal{F}_{n^{\prime}}^{\prime}\right)\left(1-\eta \mathcal{F}_{n}\right) \\
& \quad\left(\delta\left(\varepsilon_{n}-\varepsilon_{n^{\prime}}^{\prime}+\alpha^{2} \varepsilon_{p h}\right)\left[\left(N_{p h}+1\right) M_{n^{\prime}}^{\prime}-N_{p h} M_{n}\right] \varepsilon_{n}\right. \\
& \left.\quad-\delta\left(\varepsilon_{n^{\prime}}^{\prime}-\varepsilon_{n}+\alpha^{2} \varepsilon_{p h}\right)\left[\left(N_{p h}+1\right) M_{n}-N_{p h} M_{n^{\prime}}^{\prime}\right]\left(\varepsilon_{n^{\prime}}^{\prime}+\alpha^{2} \varepsilon_{p h}\right)\right) d k d k^{\prime} .
\end{aligned}
$$

By changing the variable $\varepsilon_{n}$ with $\varepsilon_{n^{\prime}}^{\prime}$ in the first term of the sum, we notice that only the $\alpha^{2}$-factor term does not vanish in this last identity, which can be rewritten as

$$
\begin{aligned}
& \sum_{n, n} \int_{\mathbb{R}^{4}} \Phi_{n, n^{\prime}}^{p h}\left(1-\eta \mathcal{F}_{n^{\prime}}^{\prime}\right)\left(1-\eta \mathcal{F}_{n}\right) \varepsilon_{p h} \delta\left(\varepsilon_{n}-\varepsilon_{n^{\prime}}^{\prime}-\alpha^{2} \varepsilon_{p h}\right)\left[N_{p h} M_{n^{\prime}}^{\prime}-\left(N_{p h}+1\right) M_{n}\right] d k d k^{\prime} \\
= & \sum_{n, n^{\prime}} \int_{\mathbb{R}^{4}} \Phi_{n, n^{\prime}}^{p h}\left(1-\eta \mathcal{F}_{n^{\prime}}^{\prime}\right)\left(1-\eta \mathcal{F}_{n}\right) \varepsilon_{p h} \delta\left(\varepsilon_{n}-\varepsilon_{n^{\prime}}^{\prime}-\alpha^{2} \varepsilon_{p h}\right) N_{p h} M_{n}\left(e^{\frac{\alpha^{2} \varepsilon_{p h}}{k_{B} T}}-e^{\frac{\alpha^{2} \varepsilon_{p h}}{k_{B} T_{L}}}\right) d k d k^{\prime},
\end{aligned}
$$


where the phonon occupation number $N_{p h}$ is defined in (2.5). Letting $\alpha \rightarrow 0$, we have that $N_{p h}\left(e^{\alpha^{2} \varepsilon_{p h} / k_{B} T}-e^{\alpha^{2} \varepsilon_{p h} / k_{B} T_{L}}\right) \rightarrow T_{L}\left(\frac{1}{T}-\frac{1}{T_{L}}\right)$. Thus

$$
W=T_{L}\left(\frac{1}{T}-\frac{1}{T_{L}}\right) \sum_{n, m} \int_{\mathbb{R}^{4}} \Phi_{n, n^{\prime}}^{p h}\left(k, k^{\prime}\right) \mathcal{F}_{n}\left(1-\eta \mathcal{F}_{n}\right) \varepsilon_{p h} \delta\left(\varepsilon_{n}-\varepsilon_{n^{\prime}}^{\prime}\right) d k d k^{\prime} .
$$

The following lemma proves that $W$ is a temperature relaxation term which relaxes $T$ to the lattice temperature $T_{L}$.

Lemma 2.10. Let $W$ be defined in (2.34). Then, we have

$$
W \cdot\left(T-T_{L}\right) \leq 0 .
$$

Proof. The proof of this result is an immediate consequence of (2.38).

2.6. Formal derivation of drift-diffusion equation. In the case where the electron-phonon scattering is dominant, after a rescaling we have $W=\frac{1}{\gamma} \widetilde{W}$ with a parameter $\gamma \ll 1$. Then equation (2.30) with expression (2.38) implies that formally in the limit $\gamma \rightarrow 0$, we have $T=T_{L}$. Then (2.29) leads to the well-known drift-diffusion model

$$
\partial_{t} \rho_{\mu}-\nabla_{x}\left(\frac{\mathbb{D}_{00}}{k_{B} T_{L}} \nabla_{x} \mu\right)=0 .
$$

Moreover, assuming $\eta=0$, the equilibrium is then given by the Boltzmann statistics. We deduce therefore from (2.36) that for $T=T_{L}$,

$$
\rho_{\mu}=2 \pi \frac{m^{*}}{\hbar^{2}} k_{B} T_{L} e^{\mu / k_{B} T_{L}} \sum_{n \in \mathbb{N}^{*}} e^{-\epsilon_{n} / k_{B} T_{L}} .
$$

Then

$$
\nabla_{x} \rho_{\mu}=\frac{1}{k_{B} T_{L}} \rho_{\mu} \nabla_{x} \mu+\rho_{\mu} \frac{\nabla_{x}\left(\sum_{n} e^{-\epsilon_{n} / k_{B} T_{L}}\right)}{\sum_{n} e^{-\epsilon_{n} / k_{B} T_{L}}}
$$

We can introduce as in [8] the effective potential energy defined by

$$
V_{s}=-k_{B} T_{L} \log \left(\sum_{n \in \mathbb{N}^{*}} e^{-\epsilon_{n} / k_{B} T_{L}}\right) .
$$

Then, we have

$$
\rho_{\mu} \nabla_{x} \mu=\left(k_{B} T_{L} \nabla_{x} \rho_{\mu}+\rho_{\mu} \nabla_{x} V_{s}\right)
$$

such that, denoting $\mathbb{D}=\mathbb{D}_{00} / \rho_{\mu}$, we recover from (2.39) the standard formulation of the drift-diffusion system [8]:

$$
\partial_{t} \rho_{\mu}-\nabla_{x} \cdot\left(\mathbb{D}\left(k_{B} T_{L} \nabla_{x} \rho_{\mu}+\rho_{\mu} \nabla_{x} V_{s}\right)\right)=0 .
$$

REMARK 2.11. We conclude the section by noting the similarities and the differences between the classical and the partially confined energy-transport model. Due to partial confinement in the $z$ direction, the electron density in the transport direction contains $T$ as factor rather than $T^{3 / 2}$ (see (2.35)). Moreover, we point out that 
the system (2.29)-(2.33) in the variables $\mu / k_{b} T,-1 / k_{b} T$ is in symmetric form, with the electric forces appearing in the diffusion coefficients through the eigenenergies $\epsilon_{n}$ (which in turn depend on the electrostatic potential). In the partially confined framework, the diffusive limiting process brought directly to a set of variables which can be interpreted as dual entropy variables (using the denomination of nonequilibrium thermodynamics $[17,27])$. In this framework, $T$ can be interpreted as electron temperature, and the variable $\mu$ as quasi-Fermi potential energy. This fact is clear considering the drift-diffusion model (2.42). Indeed, using the effective potential energy $V_{s}$ defined in (2.41), we can write the electron density (2.40) as

$$
\rho_{\mu}=2 \pi \frac{m^{*}}{\hbar^{2}} k_{B} T_{L} e^{\left(\mu-V_{s}\right) / k_{B} T_{L}},
$$

which gives the classical relation between electron density and quasi-Fermi energy. A chemical potential can then be defined as $\mu_{\text {chem }}=\mu-V_{s}$.

\section{Numerical simulation}

The device we are modelling in this work is a nanoscale Double-Gate MOSFET (Metal Oxide Semiconductor Field Effect Transistor), as in [32]. This device consists of a silicon film, characterized by two highly doped regions near the Ohmic contacts (denoted by source and drain) and an active region, called channel, with lower doping. The silicon film is sandwiched between two thin layers of silicon dioxide $\mathrm{SiO}_{2}$, each of them with a gate contact.

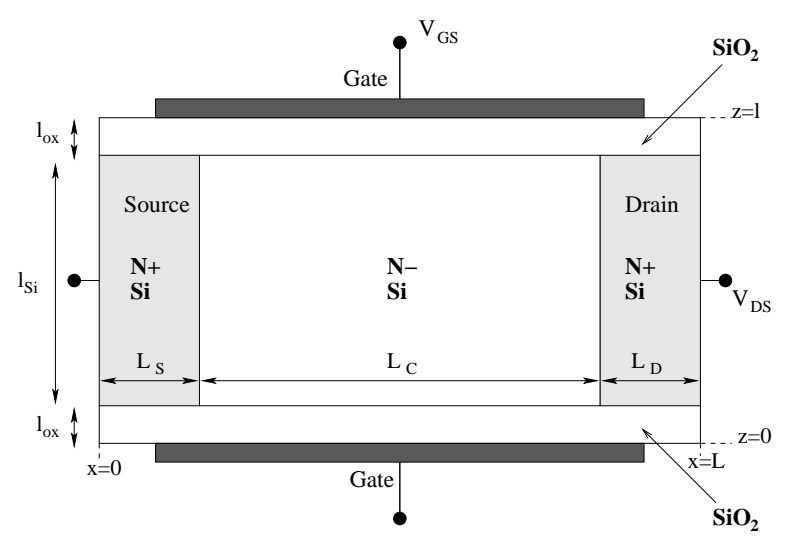

FIG. 3.1. Schematic representation of the modeled device.

We assume invariance in the $x_{2}$ direction (infinite boundary conditions), so that the problem is studied in a $\left(x_{1}, z\right)$-domain. The device occupies a region of a $2 \mathrm{D}$ domain denoted by $\Omega=[0, L] \times[0, \ell]$. A schematic representation of the device is shown in figure 3.1 .

3.1. Energy-transport-Schrödinger-Poisson system. In the following, we describe the collisional transport in the Double-Gate MOSFET, schematized in figure 3.1, by means of the energy-transport model (2.29)-(2.33). The confinement is described by the subband decomposition approach, which involves the resolution of the eigenvalue problem (2.1), taking also into account the presence of the oxide. Moreover, in order to provide explicitly computable diffusion and relaxation terms, the following physical assumptions will be used. 


\section{Assumption 3.1.}

- (H1) The cross-sections $\Phi^{0}$ and $\Phi^{p h}$ are assumed to be energy dependent functions and to have the following expression (see $[3,5,15])$ :

$$
\Phi_{n, n^{\prime}}^{0}\left(t, x, k, k^{\prime}\right)=\phi^{0}(t, x) \varepsilon_{n}^{s}, \quad \Phi_{n, n^{\prime}}^{p h}\left(t, x, k, k^{\prime}\right)=\phi^{p h}(t, x) \varepsilon_{n}^{s}
$$

with $-2<s<2$. In the physical literature, the values $s=0$ and $s=1 / 2$ have been used $[14,25,29]$.

- (H2) The electron density and the energy are assumed to be given by nondegenerate Boltzmann statistics, i.e. $\eta=0$, as in (2.36), (2.37).

Using Assumption (H1), we deduce that the diffusion matrix (2.21) has the following expression:

$$
D(t, x, \varepsilon)=\frac{1}{\phi^{0}(t, x) \varepsilon^{s} \mathcal{N}(\varepsilon)} \sum_{n \in \mathbb{N}^{*}}\left(\varepsilon-\epsilon_{n}\right)^{+} I d,
$$

where $\mathcal{N}(\varepsilon)=\max \left\{n \in \mathbb{N}^{*} / \epsilon_{n} \leq \varepsilon\right\}$ is the number of non-zero terms in the sum (see Definition 2.1). Moreover, thanks to the coarea formula in (2.38), we have

$$
W=-4 \pi^{2} \varepsilon_{p h}\left(1-\frac{T_{L}}{T}\right) \int_{\epsilon_{1}}^{+\infty} \phi^{p h} \varepsilon^{s} \mathcal{N}^{2}(\varepsilon) \mathcal{F}(\varepsilon)(1-\eta \mathcal{F}(\varepsilon)) d \varepsilon,
$$

which, under Assumption (H2), reads

$$
W=-4 \pi^{2} \varepsilon_{p h}\left(1-\frac{T_{L}}{T}\right) e^{\mu /\left(k_{B} T\right)} \int_{\epsilon_{1}}^{+\infty} \phi^{p h} \varepsilon^{s} \mathcal{N}^{2}(\varepsilon) e^{-\varepsilon /\left(k_{B} T\right)} d \varepsilon .
$$

By defining

$$
W_{0}=4 \pi^{2} \varepsilon_{p h} \int_{\epsilon_{1}}^{+\infty} \phi^{p h} \varepsilon^{s} \mathcal{N}^{2}(\varepsilon) e^{-\varepsilon /\left(k_{B} T\right)} d \varepsilon
$$

we have the compact expression

$$
W=-W_{0}\left(1-\frac{T_{L}}{T}\right) e^{\mu /\left(k_{B} T\right)} .
$$
by

Assumption (H2) implies also that the density of charge carriers $N_{e}(t, x, z)$ is given

$$
N_{e}=\sum_{n \in \mathbb{N}^{*}} \int_{\mathbb{R}^{2}} \mathcal{F}_{\mu, T}\left(t, x, \frac{|k|^{2} \hbar^{2}}{2 m^{*}}+\epsilon_{n}\right) d k\left|\chi_{n}\right|^{2}=\frac{2 \pi m^{*} k_{B} T}{\hbar^{2}} \sum_{n \in \mathbb{N}^{*}} e^{\left(\mu-\epsilon_{n}\right) / k_{B} T}\left|\chi_{n}\right|^{2} .
$$

Finally, the coupled subband energy-transport model under Assumption 3.1 is given by: Find $\mu(t, x), T(t, x),\left(\epsilon_{n}(t, x), \chi_{n}(t, x)\right)$ for $n \geq 1$, and $V(t, x, z)$ such that

$$
\begin{gathered}
\partial_{t} \rho_{\mu, T}-\nabla_{x} \cdot J_{1}=0, \quad \text { in }(0, L), \\
\partial_{t}\left(\rho \mathcal{E}_{\mu, T}\right)+\sum_{n \in \mathbb{N}^{*}} 2 \pi \frac{m^{*}}{\hbar^{2}} \partial_{t} \epsilon_{n} k_{B} T(t, x) e^{\left(\mu-\epsilon_{n}\right) / k_{B} T}-\nabla_{x} \cdot J_{2}=W, \quad \text { in }(0, L),
\end{gathered}
$$




$$
\begin{aligned}
& \left\{\begin{array}{l}
-\frac{\hbar^{2}}{2} \frac{d}{d z}\left(\frac{1}{m^{*}} \frac{d}{d z} \chi_{n}\right)-\left(e V-U_{c}\right) \chi_{n}=\epsilon_{n} \chi_{n}, \quad \text { in }(0, \ell) \\
\chi_{n}(t, x, \cdot) \in H_{0}^{1}(0, \ell), \quad \int_{0}^{\ell} \chi_{n} \chi_{n^{\prime}} d z=\delta_{n n^{\prime}}
\end{array}\right. \\
& \operatorname{div}_{x, z}\left(\varepsilon_{R} \nabla_{x, z} V\right)=\frac{e}{\varepsilon_{0}}\left(\frac{2 \pi m^{*} k_{B} T}{\hbar^{2}} \sum_{n \in \mathbb{N}^{*}} e^{\left(\mu-\epsilon_{n}\right) / k_{B} T}\left|\chi_{n}\right|^{2}-N_{D}\right), \quad \text { in } \Omega,
\end{aligned}
$$

where the expressions of $\rho_{\mu, T}$ and $\mathcal{E}_{\mu, T}$ with respect to the unknowns are given in (2.36)-(2.37). In (3.7) the effective mass $m^{*}$ takes different values in the $S i$ and in the $\mathrm{SiO}_{2}$ domain. Moreover, $U_{c}$ represents a given potential barrier between the silicon and the oxide. The currents $J_{1}$ and $J_{2}$ are given by the expressions

$$
\begin{aligned}
& J_{1}=\mathbb{D}_{00} \nabla_{x}\left(\frac{\mu}{k_{B} T}\right)-\mathbb{D}_{01} \nabla_{x}\left(\frac{1}{k_{B} T}\right), \\
& J_{2}=\mathbb{D}_{10} \nabla_{x}\left(\frac{\mu}{k_{B} T}\right)-\mathbb{D}_{11} \nabla_{x}\left(\frac{1}{k_{B} T}\right),
\end{aligned}
$$

where, under Assumption 3.1, the diffusion coefficients are given by

$$
\mathbb{D}_{i j}=\frac{1}{\phi^{0}} \sum_{n \in \mathbb{N}^{*}} \int_{\epsilon_{n}}^{+\infty} \frac{\varepsilon^{i+j-s}\left(\varepsilon-\epsilon_{n}\right)}{\mathcal{N}(\varepsilon)} e^{(\mu-\varepsilon) / k_{B} T} d \varepsilon
$$

The relaxation term $W$ is given by (3.4).

This system is complemented with initial and boundary conditions. In particular, at the ohmic contacts and at the gate (see figure 3.1), we will impose Dirichlet boundary conditions for the potential; otherwise we fix homogeneous Neumann boundary conditions, which model isolating conditions.

$$
\begin{aligned}
V(x, z) & =V_{\text {Gate }}, & & \text { for } z \in\{0, \ell\}, x \in \text { Gate, } \\
V(x, z) & =V_{D}, & & \text { for } x \in\{0, L\}, z \in(0, \ell), \\
\frac{\partial V}{\partial \nu} & =0, & & \text { elsewhere, }
\end{aligned}
$$

where $\nu$ is the outward unit normal. Since the transport occurs only in the longitudinal direction, we just have to impose boundary conditions in $x=0$ and $x=L$ for $\mu$ and $T$. The temperature is assumed to be at the lattice temperature $T_{L}$, thus

$$
T(x)=T_{L}, \quad \text { for } x \in\{0, L\} .
$$

Then, we consider that the surface density of the charge carriers is almost constant near the frontiers $x=0$ and $x=L$ and given by $N_{s}^{b}$. The surface density being the integral over $z$ of the total density $\left(N_{s}^{b}=N^{+} \times \ell_{S i}\right)$, we deduce

$$
\mu(x)=\mu_{b}:=k_{B} T_{L} \log \left(\frac{N_{s}^{b} \hbar^{2}}{2 \pi m k_{B} T_{L} \sum_{n} e^{-\epsilon_{n} / k_{B} T_{L}}}\right), \quad \text { for } x \in\{0, L\} .
$$

3.2. Stationary system. Let us introduce the notations

$$
u=\frac{\mu}{k_{B} T}, \quad v=-\frac{1}{k_{B} T} .
$$


Then we can rewrite the expressions of the current (3.9)-(3.10) as

$$
\begin{aligned}
& J_{1}=\mathbb{D}_{00}(u, v) \nabla_{x} u+\mathbb{D}_{01}(u, v) \nabla_{x} v, \\
& J_{2}=\mathbb{D}_{10}(u, v) \nabla_{x} u+\mathbb{D}_{11}(u, v) \nabla_{x} v,
\end{aligned}
$$

where the diffusion coefficients are given by

$$
\mathbb{D}_{i j}(u, v)=\frac{1}{\phi^{0}} \sum_{n \in \mathbb{N}^{*}} \int_{\epsilon_{n}}^{+\infty} \frac{\varepsilon^{i+j-s}\left(\varepsilon-\epsilon_{n}\right)}{\mathcal{N}(\varepsilon)} e^{u+\varepsilon v} d \varepsilon .
$$

We define the relaxation coefficient in the same way:

$$
W_{0}(u, v)=4 \pi^{2} \varepsilon_{p h} \int_{\epsilon_{1}}^{+\infty} \phi^{p h} \varepsilon^{s} \mathcal{N}^{2}(\varepsilon) e^{(\varepsilon-\mu) v} d \varepsilon .
$$

Then, the stationary version of the energy-transport subband system (3.5)-(3.6) in variable $u$ and $v$ writes

$$
\begin{gathered}
-\nabla_{x}\left(\mathbb{D}_{00}(u, v) \nabla_{x} u+\mathbb{D}_{01}(u, v) \nabla_{x} v\right)=0, \\
-\nabla_{x}\left(\mathbb{D}_{10}(u, v) \nabla_{x} u+\mathbb{D}_{11}(u, v) \nabla_{x} v\right)=-W_{0}(u, v)\left(1+k_{B} T_{L} v\right) .
\end{gathered}
$$

Boundary conditions (3.14)-(3.15) become

$$
v(x)=v_{b}:=-\frac{1}{k_{B} T_{L}}, \quad \text { for } x \in\{0, L\},
$$

and

$$
u(x)=u_{b}:=\log \left(\frac{N_{s}^{b} \hbar^{2}}{2 \pi m k_{B} T_{L} \sum_{n} e^{-\epsilon_{n} / k_{B} T_{L}}}\right), \quad \text { for } x \in\{0, L\} .
$$

3.3. Numerical approach for the energy-transport system. We introduce a partition of $[0, L]$ with nodes $x_{i}, i=0, \cdots, N_{x}$, and a partition of $[0, \ell]$ with nodes $z_{j}, j=0, \cdots, N_{z}$. We assume that the partitions are uniform and denote $h=x_{i}-x_{i-1}$. Then, we mesh the domain $[0, L] \times[0, \ell]$ with rectangular triangles using the nodes $\left(x_{i}, z_{j}\right)$ previously defined. The Schrödinger equations and the Poisson equation are discretized with conforming $P^{1}$ finite elements. We shall use the notation $q=\left(q_{i}\right)$, $i=0, \cdots, N_{x}$ for a vector in $\mathbb{R}^{N_{x}+1}$ associated with the nodal values in $x_{i}, i=0, \cdots, N_{x}$.

We consider here in detail the discretization scheme for the equations governing $u$ and $v$, assuming first that the eigenenergies $\epsilon_{n}$ are known. Using the notations

$$
U=(u, v)^{\top}, \quad \mathcal{J}=\left(J_{1}, J_{2}\right)^{\top}, \quad W(U)=\left(0, W_{0}(u, v)\left(1+k_{B} T_{L} v\right)\right)^{\top},
$$

equations (3.17)-(3.18) and (3.21)-(3.22) can be written in compact form as

$$
\mathcal{J}=\mathbb{D}(U) \nabla_{x} U, \quad \nabla_{x} \cdot \mathcal{J}=W(U) .
$$

Denoting by $U_{i}$ an approximation of $U\left(x_{i}\right)$, we take the piecewise constant approximation of $U$ given, in the interval $I_{i}:=\left(x_{i-1}, x_{i}\right)$, by

$$
\bar{U}_{i}=\frac{U_{i-1}+U_{i}}{2}
$$


and define the piecewise constant diffusion coefficients and relaxation terms as

$$
\overline{\mathbb{D}}_{k l}=\mathbb{D}_{k l}(\bar{U}), \quad \text { for } k, l=0,1, \quad \bar{W}=W(\bar{U}) .
$$

We are going to use a mixed finite element discretization of lowest order in hybridized form [10] (see also $[20,21]$ for applications to ET). Let us introduce the following finite dimensional spaces:

$$
\begin{aligned}
X_{h} & =\left\{\sigma \in L^{2}((0, L)): \sigma(x)=a_{i}+b_{i}\left(x-x_{B_{i}}\right) \text { in } I_{i}, i=1, \ldots, N_{x}\right\}, \\
Y_{h} & =\left\{\xi \in L^{2}((0, L)): \xi \text { is constant in } I_{i}, i=1, \ldots, N_{x}\right\} \\
Z_{h, \chi} & =\left\{q=\left(q_{i}\right), i=0, \ldots, N_{x}, \text { s.t. } q_{0}=\chi(0), q_{N_{x}}=\chi(L)\right\}
\end{aligned}
$$

where $x_{B_{i}}$ denotes the central point of the interval $I_{i}$, and $\chi$ is prescribed.

Then, the mixed-hybrid formulation of (3.23) reads as follows: Find $\mathcal{J}_{h} \in X_{h}^{2}$, $P_{h} \in Y_{h}^{2}$, and $U_{h} \in Z_{h, u_{b}} \times Z_{h, v_{b}}$ such that

$$
\begin{aligned}
\sum_{i=1}^{N_{x}}\left(\int_{I_{i}} \phi_{h} \cdot \mathbb{D}\left(\bar{U}_{i}\right)^{-1} \mathcal{J}_{h} d x+\int_{I_{i}} P_{h} \cdot \nabla_{x} \phi_{h} d x-\left[\phi_{h} \cdot U_{h}\right]_{x_{i-1}}^{x_{i}}\right) & =0 \\
\sum_{i=1}^{N_{x}}\left(\int_{I_{i}} \Psi_{h} \cdot \nabla_{x} \mathcal{J}_{h} d x-\int_{I_{i}} W\left(\bar{U}_{i}\right) \cdot \Psi_{h} d x\right) & =0, \\
-\sum_{i=1}^{N_{x}}\left[\mu_{h} \cdot \mathcal{J}_{h}\right]_{x_{i-1}}^{x_{i}} & =0
\end{aligned}
$$

for all $\phi_{h} \in X_{h}^{2}, \Psi_{h} \in Y_{h}^{2}$, and $\mu_{h} \in Z_{h, 0}^{2}$. Equation (3.25) is derived from the weak formulation of the first equations in (3.23); (3.26) comes from the weak form of the second equations in (3.23); and finally, (3.27) imposes the continuity of the currents at the nodes.

Thanks to the discontinuity of the spaces $X_{h}$ and $Y_{h}$, we can apply static condensation in order to reduce the size of the discrete system and obtain an algebraic system for the variable $U_{h}$ only. More precisely, choosing first the test functions

$$
\phi_{h}=\left\{\begin{array}{l}
(1,0)^{\top} \text { in } I_{i}, \\
(0,0)^{\top} \text { elsewhere, }
\end{array} \text { and } \phi_{h}=\left\{\begin{array}{l}
(0,1)^{\top} \text { in } I_{i}, \\
(0,0)^{\top} \text { elsewhere },
\end{array}\right.\right.
$$

in (3.25), and then analogously choosing $\Psi_{h}$ in (3.26), we obtain the piecewise linear (discrete) current

$$
\mathcal{J}_{h \mid I_{i}}=\mathbb{D}\left(\bar{U}_{i}\right) \frac{U_{i}-U_{i-1}}{h}+W\left(\bar{U}_{i}\right)\left(x-x_{B_{i}}\right) .
$$

Imposing continuity at the nodes (through (3.27)) we obtain the final system

$$
-\mathbb{D}\left(\bar{U}_{i}\right) U_{i-1}+\left(\mathbb{D}\left(\bar{U}_{i}\right)+\mathbb{D}\left(\bar{U}_{i+1}\right)\right) U_{i}-\mathbb{D}\left(\bar{U}_{i+1}\right) U_{i+1}=-\frac{h^{2}}{2}\left(W\left(\bar{U}_{i}\right)+W\left(\bar{U}_{i+1}\right)\right),
$$

for $i=1, \ldots, N_{x}-1$. We point out explicitly that, since the first component of $W(U)$ is null, the approximation of the current $J_{1}$ is piecewise constant (see (3.28)) and that, thanks to (3.27), it is indeed globally constant.

System (3.29) forms a non-linear system in the unknown $(u, v)$ that can be solved using a Newton algorithm. We point out that the Jacobian corresponding to this 
non-linear system can be easily computed after noticing that, from the expressions (3.24) and (3.19), we have

$$
\frac{\partial \overline{\mathbb{D}}_{k \ell}^{i}}{\partial u_{i}}=\frac{\partial \overline{\mathbb{D}}_{k \ell}^{i}}{\partial u_{i-1}}=\frac{1}{2} \overline{\mathbb{D}}_{k \ell}^{i}, \quad \frac{\partial \overline{\mathbb{D}}_{k \ell}^{i}}{\partial v_{i}}=\frac{\partial \overline{\mathbb{D}}_{k \ell}^{i}}{\partial v_{i-1}}=\frac{1}{2} \overline{\mathbb{D}}_{k \ell+1}^{i},
$$

and that similar relations hold for the partial derivatives of $\bar{W}^{i}$.

REMARK 3.2. The expression (3.19) is not practical for numerical purposes. However, with the definition $\mathcal{N}(\varepsilon)=\max \left\{n \in \mathbb{N}^{*}: \epsilon_{n} \leq \varepsilon\right\}$, we have

$$
\begin{aligned}
\mathbb{D}_{i j}(u, v) & =\frac{1}{\phi^{0}} \sum_{n=1}^{+\infty} \sum_{m=n}^{+\infty} \int_{\epsilon_{m}}^{\epsilon_{m+1}} \frac{\left(\varepsilon-\epsilon_{n}\right) \varepsilon^{i+j-s}}{m} e^{u+\varepsilon v} d \varepsilon \\
& =\frac{1}{\phi^{0}} \sum_{m=1}^{+\infty} \int_{\epsilon_{m}}^{\epsilon_{m+1}}\left(\varepsilon-\frac{\sum_{n=1}^{m} \epsilon_{n}}{m}\right) \varepsilon^{i+j-s} e^{u+\varepsilon v} d \varepsilon,
\end{aligned}
$$

by interchanging the sums over $m$ and $n$. We can rewrite this as

$$
\begin{aligned}
\mathbb{D}_{i j}(u, v)=\frac{1}{\phi^{0}} \int_{\epsilon_{1}}^{+\infty} \varepsilon^{i+j+1-s} e^{u+\varepsilon v} d \varepsilon & \\
& \quad+\frac{1}{\phi^{0}} \sum_{m=1}^{+\infty}\left(\frac{\sum_{n=1}^{m-1} \epsilon_{n}}{m-1}-\frac{\sum_{n=1}^{m} \epsilon_{n}}{m}\right) \int_{\epsilon_{m}}^{+\infty} \varepsilon^{i+j-s} e^{u+\varepsilon v} d \varepsilon,
\end{aligned}
$$

with the convention that $\sum_{n=1}^{m-1} \epsilon_{n} /(m-1)=0$ for $m=1$. Then, in the actual numerical computation, we can get an accurate approximation of $\mathbb{D}_{i j}$ by truncating the infinite sum to a finite number of eigenmodes. In fact, $\left(\epsilon_{n}\right)_{n}$ forms an increasing sequence going to $+\infty$. Thus, since $v<0$, we have that $\left(e^{\epsilon_{n} v}\right)_{n}$ fast decreases to 0 .

3.4. Algorithm. We are now ready to describe the algorithm used for the numerical resolution of the stationary subband energy-transport model. The first step of the algorithm is the computation of the thermal equilibrium solution, with no applied drain-source bias. In this case the temperature and the Fermi level are constant along the device, and therefore the problem reduces to solving the Poisson equation (3.8) for a given temperature and Fermi level, which can be computed thanks to the boundary conditions. The computed potential at thermal equilibrium is used as starting data for the following Gummel [22] iteration process:

1. Let $V_{\text {old }}$ be a given potential.

2. We solve the eigenvalue problem (3.7) on each vertical slice of the domain by diagonalization of the Hamiltonian. Therefore we obtain the set $\left\{\chi_{n}\left(x_{i}, z_{j}\right)\right\}$ and $\left\{\epsilon_{n}\left(x_{i}\right)\right\}$.

3. We implement the Newton procedure which has been described above for the computation of $(u, v)$.

4. We compute the density of charge carriers corresponding to the right hand side of (3.8):

$$
N_{e}=\sum_{n \in \mathbb{N}^{*}} 2 \pi \frac{m^{*}}{\hbar^{2}} \frac{e^{u}}{v} e^{\epsilon_{n} v}\left|\chi_{n}\right|^{2} .
$$

We are then able to solve the Poisson equation (3.8) with boundary condition (3.11). Indeed, due to the strong coupling of the entire system, the simple 


\begin{tabular}{|c||c||c||c|}
\hline Parameter & Value & Length & Value \\
\hline \hline$N^{+}$ & $10^{26} \mathrm{~m}^{-3}$ & $L_{S}$ & $10 \mathrm{~nm}$ \\
\hline$N^{-}$ & $10^{21} \mathrm{~m}^{-3}$ & $L_{C}$ & $30 \mathrm{~nm}$ \\
\hline$U_{c}$ & $3 \mathrm{eV}$ & $L_{D}$ & $10 \mathrm{~nm}$ \\
\hline$\varepsilon_{R}[\mathrm{Si}]$ & 11.7 & $\ell_{o x}$ & $3 \mathrm{~nm}$ \\
\hline$\varepsilon_{R}\left[\mathrm{SiO}_{2}\right]$ & 3.9 & $\ell_{S i}$ & $5 \mathrm{~nm}$ \\
\hline
\end{tabular}

TABLE 3.1. Table of the main physical values.

resolution of equation (3.8) does not provide a converging algorithm. Following [12] a Gummel iteration algorithm is used, which amounts to computing the new potential $V_{\text {new }}$ by solving the following modified Poisson equation:

$$
\operatorname{div}\left(\varepsilon_{R} \nabla V_{\text {new }}\right)+\frac{e}{\varepsilon_{0}} N_{e} \frac{V_{\text {new }}}{V_{\text {ref }}}=\frac{e}{\varepsilon_{0}}\left(N_{e}\left(1-\frac{V_{\text {old }}}{V_{\text {ref }}}\right)-N_{D}\right), \quad \text { in } \Omega,
$$

with $V_{\text {ref }}=k_{B} T_{L} / e$.

5. We repeat the last three steps until the quantity $\left\|V_{\text {old }}-V_{\text {new }}\right\|_{L^{\infty}}$ becomes sufficiently small. Once the convergence is reached, we increment the applied drain-source bias $V_{D S}$ of $0.02 \mathrm{~V}$ and start a new iteration.

3.5. Numerical results. In this section we present and comment on the numerical results. The modeled device is schematized in figure 3.1 and the physical values are chosen as the ones in [32], which are recalled in table 3.1. We take $N_{x}=50$ points in the transport direction and $N_{z}=50$ in the confined direction for all the tests. The results are presented for $s=\frac{1}{2}$ in (3.1), which corresponds to the so-called Chen model [14].
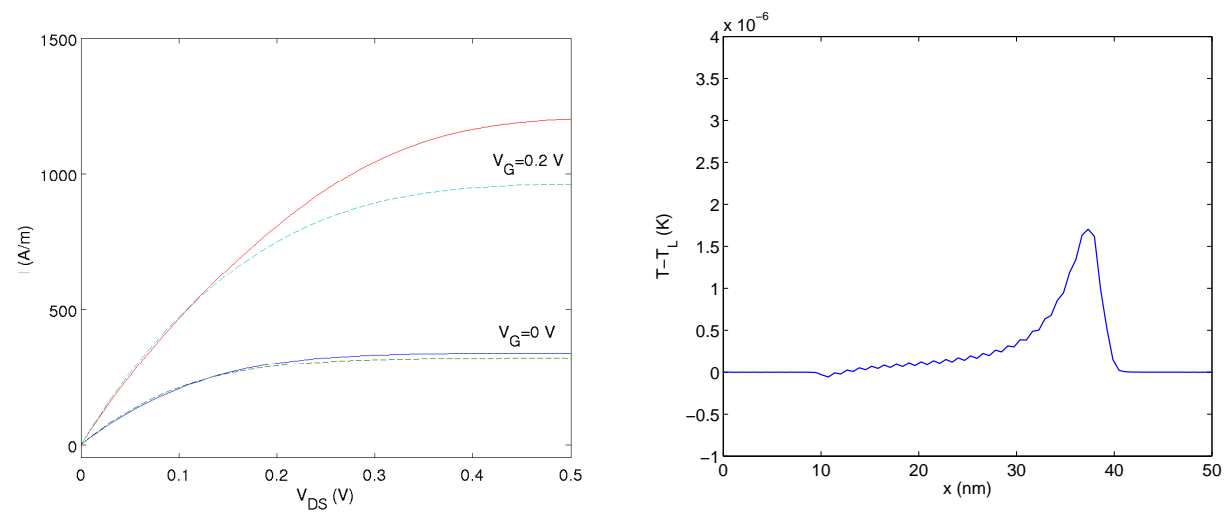

FIG. 3.2. Left: $I-V_{D S}$ characteristics for $V_{G}=0 \mathrm{~V}$ and $V_{G}=0.2 \mathrm{~V}$. The solid line corresponds to $\phi^{p h}=10^{-4} / \phi^{0}$, and the dashed line corresponds to $\phi^{p h}=10^{5} / \phi^{0}$, which is a good approximation to the drift-diffusion model. Right: Temperature $T-T_{L}$ in the device for $\phi^{p h}=10^{5} / \phi^{0}$. We see that the variations of the temperature are of order $10^{-6} \mathrm{~K}$.

Some other physical coefficients should be determined. The effective mass is $m^{*}=0.19 m_{e}$ with $m_{e}$ the electron mass, the lattice temperature is $T_{L}=300 \mathrm{~K}$, and the scattering coefficient for the elastic collisions is the one used in [32] $\phi^{0}=\frac{1}{\mu^{0} n_{i}}$, 

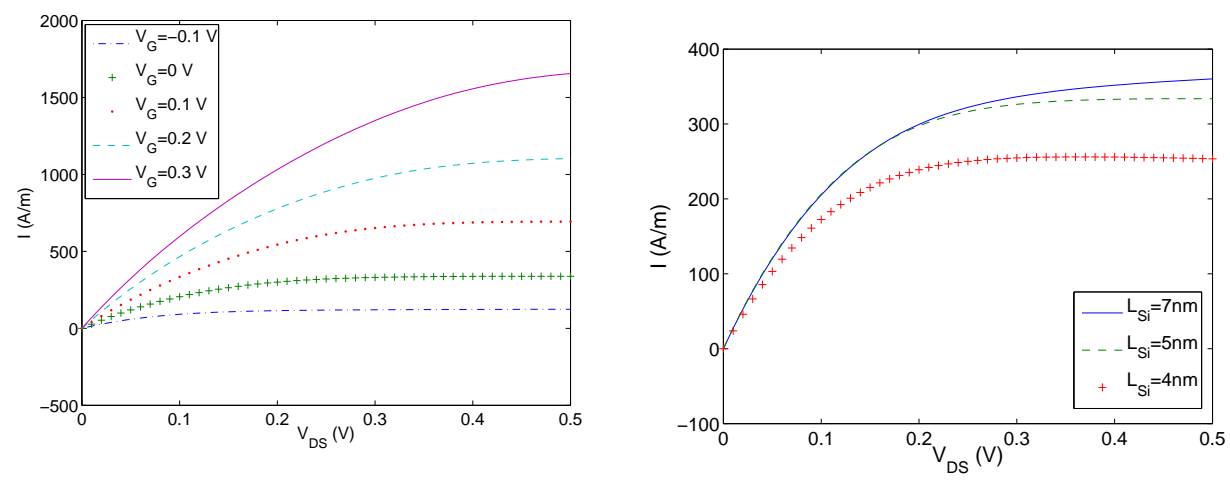

FIG. 3.3. $I-V_{D S}$ characteristics for different Gate voltages $V_{G}$ with $\ell_{S i}=5 \mathrm{~nm}$ (left), and for different width of the Silicon in the DG-MOSFET with $V_{G}=0 \mathrm{~V}$ (right).
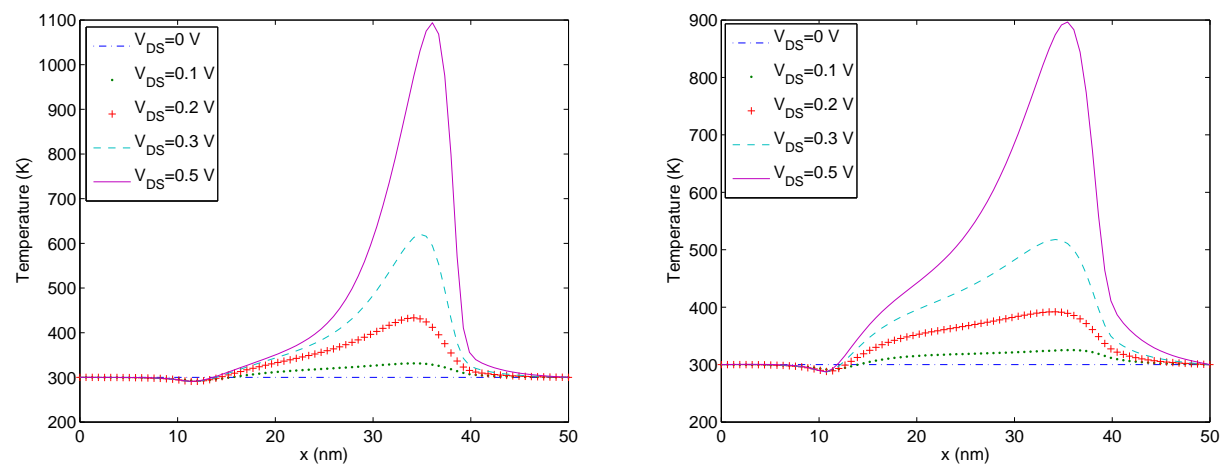

FIG. 3.4. Variation of the temperature with respect to $V_{D S}$ for a Gate voltage $V_{G}=0 V$ (left) and $V_{G}=0.2 \mathrm{~V}$ (right).

where the low field mobility is taken as $\mu^{0}=0.12 \mathrm{~m}^{2} V^{-1} \mathrm{~s}^{-1}$ and the intrinsic density is given by $n_{i}=10^{10} \mathrm{~m}^{-2}$. We have to fix the value of the scattering coefficient $\phi^{p h}$ for the electron-phonon interaction. As noticed in Subsection 2.6, when $\phi^{p h} \rightarrow+\infty$, the model converges formally to the subband drift-diffusion system presented in $[8,32]$. It is then interesting to compare the numerical results for large and small values of $\phi^{p h}$. Figure 3.2 displays the $I-V_{D S}$ characteristics for $\phi^{p h}=10^{-4} / \phi^{0}$ and $\phi^{p h}=10^{5} / \phi^{0}$. As expected and as noticed in [2], the energy-transport model gives higher currents compared with the drift-diffusion model, due to the electron velocity overshoot within the channel. Figure 3.2 (right) displays the curve $T-T_{L}$ with respect to the transport direction $x$, where $T$ denotes the temperature, for $\phi^{p h}=10^{5} / \phi^{0}$. It confirms that we are in the drift-diffusion regime, where the temperature is constant.

In the rest of the section, we present the results only for $\phi^{p h}=10^{-4} / \phi^{0}$, which corresponds to the energy-transport regime. Figure 3.3 displays the computed current vs drain-source applied bias characteristics with this chosen value. We present in figure 3.3 (left) the characteristics for different numerical values of the Gate voltage $V_{G}$ and with $\ell_{S i}=5 \mathrm{~nm}$. In figure 3.3 (right), we display the characteristics for $V_{G}=0$ and 

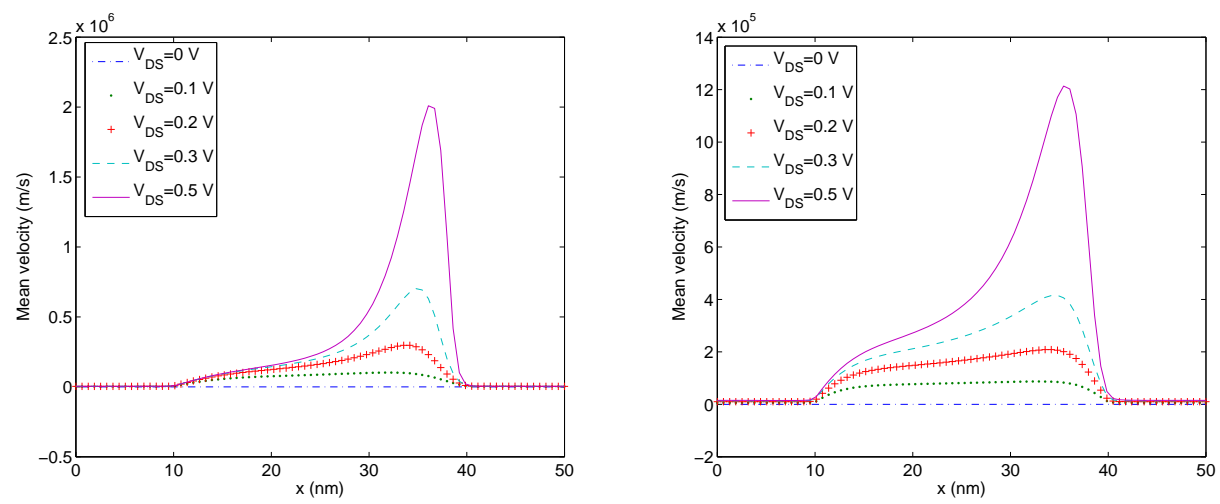

FIG. 3.5. Mean velocity for different drain-source potentials $V_{D S}$ and for $V_{G}=0 V$ (left) and $V_{G}=0.2 \mathrm{~V}$ (right).
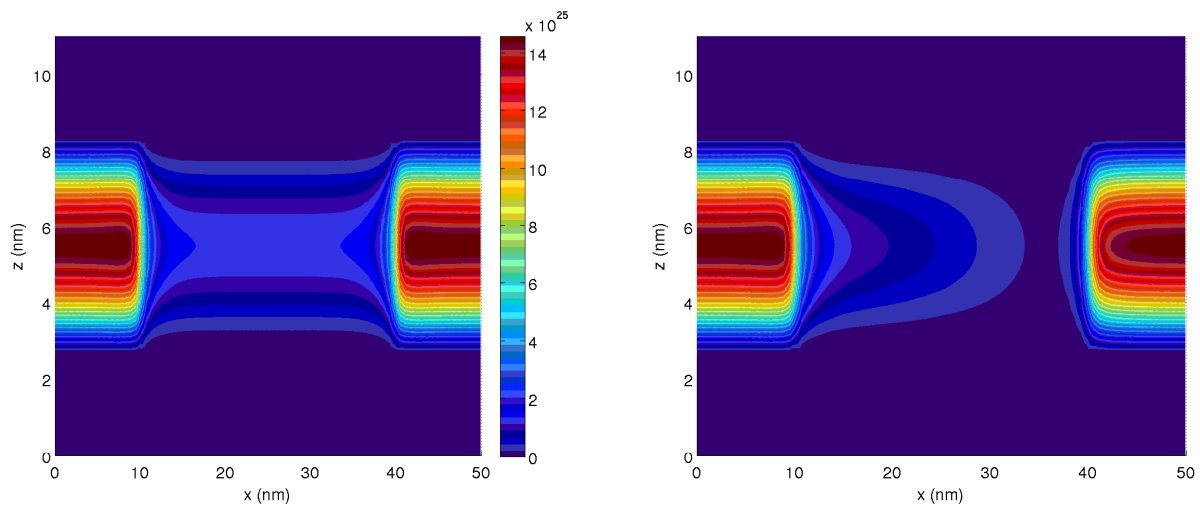

FIG. 3.6. Density of electrons in the device for $V_{D S}=0 \mathrm{~V}$ (left) and $V_{D S}=0.5 \mathrm{~V}$ (right); in this simulation we take $V_{G}=0.2 \mathrm{~V}$.

for different geometry of the devices : $\ell_{S i}=4,5$, or $7 \mathrm{~nm}$. These characteristics are comparable to the one obtained in $[2,31,32]$. We present in figure 3.4 the evolution of the temperature in the device with respect to the drain-source voltage for two different values of the Gate voltage. Figure 3.5 displays the evolution of the mean velocity, defined by $J_{1} /\left(q \rho_{\mu, T}\right)$, where the one dimensional density is given in (2.36) and the current in (3.17). As expected, we notice an overshoot of the velocity at the frontier between the channel and the drain for high values of $V_{D S}$.

We plot in figure 3.6 the shape of the density in the device for two different drainsource voltages. For $V_{D S}=0 \mathrm{~V}$, we are at equilibrium and the density is symmetric in the device. For $V_{D S}=0.5 \mathrm{~V}$, we notice transport of the charge carriers in the device.

\section{Conclusion}

A coupled quantum-classical model has been obtained for describing the transport of a partially confined electron gas. In a subband decomposition framework, the transport model is obtained by means of diffusive approximation from adiabatic quantum-kinetic models. The final system in the transport direction is obtained 
through two steps. First, under the assumption of dominant elastic scattering, a SHE system is derived (referring to [7]). Then, under dominant electron-electron collisions, an energy transport model is given, obtaining diffusion coefficients well suited for numerical purposes and with a relaxation term taking into account the electron-phonon interactions. In particular, with energy dependent cross-section of the collision operator explicit expression of the diffusion coefficients and of the relaxation term is derived and used for the numerical simulation of transport in a Double-Gate MOSFET. In the limit of large electrons-phonons collisions, we recover the model of [32]. We point out that a saturation of the current is observed without resorting to mobility modeling as done in [32].

Acknowledgment. The authors acknowledge partial support from the Galilée Project No. 25992ND of the Hubert Curien program: "Modèles numériques du transport collisionnel dans des dispositifs nano-électroniques".

\section{REFERENCES}

[1] N.W. Ashcroft and N.D. Mermin, Solid State Physics, Brooks Cole Ed., 1976.

[2] G. Baccarani and S. Reggiani, A compact double-gate MOSFET model comprising quantummechanical and nonstatic effects, IEEE Trans. Electron Devices, 46(8), 1656-1666, 1999.

[3] N. Ben Abdallah and P. Degond, On a hierarchy of macroscopic models for semiconductors, J. Math. Phys., 37(7), 3308-3333, 1996.

[4] N. Ben Abdallah, P. Degond, and S. Génieys, An energy transport model for the semiconductors derived from the Boltzmann equation, J. Stat. Phys., 84(1-2), 205-231, 1996.

[5] N. Ben Abdallah, P. Degond, P. Markowich, and C. Schmeiser, High field approximation of the spherical harmonic expansion model for semiconductors, Z. Angew. Math. Phys., 52, 201-230, 2001.

[6] N. Ben Abdallah, L. Desvillettes, and S. Génieys, On the convergence of the Boltzmann equation for semiconductors towards the energy transport model, J. Stat. Phys., 98(3-4), 835-870, 2000.

[7] N. Ben Abdallah, F. Méhats, and C. Negulescu, Adiabatic quantum-fluid transport models, Commun. Math. Sci., 4(3), 621-650, 2006.

[8] N. Ben Abdallah, F. Méhats, and N. Vauchelet, Diffusive transport of partially quantized particles : Existence, uniqueness and long time behaviour, Proc. Edinb. Math. Soc., 49, 513-549, 2006.

[9] J.P. Bourgade, P. Degond, F. Méhats, and C. Ringhofer, On quantum extensions to classical spherical harmonics expansion/Fokker-Planck models, J. Math. Phys., 47(4), 043302, 2006.

[10] F. Brezzi and M. Fortin, Mixed and Hybrid Finite Element Methods, Springer Series in Computational Mathematics, Springer-Verlag, New York, 15, 1991.

[11] F. Brezzi, L. Marini, and P. Pietra, Two-dimensional exponential fitting and applications to drift-diffusion models, SIAM J. Num. Anal., 26, 1342-1355, 1989.

[12] P. Caussignac, B. Zimmermann, and R. Ferro, Finite element approximation of electrostatic potential in one dimensional multilayer structures with quantized electronic charge, Computing, 45, 251-264, 1990.

[13] C. Chainais-Hillairet, Discrete duality finite volume schemes for two-dimensional drift-diffusion and energy-transport models, Internat. J. Numer. Methods Fluids, 59(3), 239-257, 2009.

[14] D. Chen, E. Kan, U. Ravaioli, C. Shu, and R. Dutton, An improved energy transport model including nonparabolicity and non-maxwellian distribution effects, IEEE Electr. Dev. Letters, 13, 26-28, 1992.

[15] P. Degond, A. Jüngel, and P. Pietra, Numerical discretization of energy-transport models for semiconductors with non-parabolic band structure, SIAM J. Sci. Comput., 22, 986-1007, 2000.

[16] P. Degond, F. Méhats, and C. Ringhofer, Quantum energy-transport and drift-diffusion models, J. Stat. Phys., 118(3-4), 625-665, 2005.

[17] S. De Groot and P. Mazur, Nonequilibrium Thermodynamics, Dover Publications, New York, 1984.

[18] M. Fournié, Numerical discretization of the energy-transport model for semiconductors, Appl. Math. Letters, 15(6), 721-726, 2002. 
[19] C. Fürst, A. Leitenstorfer, A. Laubereau, and R. Zimmermann, Quantum kinetic electronphonon interaction in GaAs: Energy nonconserving scattering events and memory effects, Phys. Rev. Lett., 78, 3733-3736, 1997.

[20] S. Gadau and A. Jüngel, A three-dimensional mixed finite-element approximation of the semiconductor energy-transport equations, SIAM J. Sci. Comput., 31(2), 1120-1140, 2008/09.

[21] S. Gadau, A. Jüngel, and P. Pietra, A mixed finite-element scheme of a semiconductor energytransport model using dual entropy variables, Hyperbolic Problems: Theory, Numerics and Applications. I, Yokohama Publ., Yokohama, 139-146, 2006.

[22] H.K. Gummel, A self-consistent iterative scheme for one-dimensional steady state transistor calculations, IEEE Trans. on Elec. Dev., 11(10), 455-465, 1964.

[23] S. Holst, A. Jüngel, and P. Pietra, A mixed finite-element discretization of the energy-transport model for semiconductors, SIAM J. Sci. Comput., 24(6), 2058-2075, 2003.

[24] J.W. Jerome and C.W. Shu, Energy transport systems for semiconductors: Analysis and simulation, World Congress of Nonlinear Analysis, Vol. I-IV. de Gruyter: Berlin, 3835-3846, 1996.

[25] A. Jüngel, Transport Equations for Semiconductors, Lectures Notes in Physics, Springer, Berlin, 773, 2009.

[26] A. Jüngel and D. Matthes, A derivation of the isothermal quantum hydrodynamic equations using entropy minimization, ZAMM Z. Angew. Math. Mech., 85(11), 806-814, 2005.

[27] H. Kreuzer, Nonequilibrium Thermodynamics and its Statistical Fundation, Clarondon Press, Oxford, 1981.

[28] C. Lab and P. Caussignac, An energy-transport model for semiconductor heterostrucures devices: Application to AlGaAs/GaAs Modfets, COMPEL, 18, 61-76, 1999.

[29] E. Lyumkis, B. Polsky, A. Shur, and P. Visocky, Transient semiconductor device simulation including energy balance equation, COMPEL, 11, 311-325, 1992.

[30] A. Marrocco and P. Montarnal, Simulation de modèles "Energy Transport" à l'aide des Éléments Finis Mixtes, C.R. Acad. Sci. Paris Sér. I Math., 323, 1996.

[31] C. Negulescu, N. Ben Abdallah, E. Polizzi, and M. Mouis, Simulation schemes in 2D nanoscale MOSFETs: A WKB based method, J. Comput. Electron., 3(3-4), 397-400, 2005.

[32] P. Pietra and N. Vauchelet, Modeling and simulation of the diffusive transport in a nanoscale Double-Gate MOSFET, J. Comput. Electron., 7, 52-65, 2008.

[33] E. Polizzi and N. Ben Abdallah, Subband decomposition approach for the simulation of quantum electron transport in nanostructures, J. Comput. Phys., 202, 150-180, 2005.

[34] L. Reggiani (ed.), Hot Electon Transport in Semiconductors, Springer, Berlin, 1985.

[35] C. Ringhofer, An entropy-based finite difference method for the energy transport system, Math. Models Meth. Appl. Sci., 11, 769-796, 2001.

[36] J. Schilp, T. Kuhn, and G. Mahler, Electron-phonon quantum kinetics in pulse excited semiconductors: Memory and renormalization effects, Phys. Rev. B, 50, 5435-5447, 1994. 